\title{
Räume und Orte der Zeit. Architektonische Utopien und filmische Heterotopien
}

In dieser Untersuchung werden vornehmlich die verschiedenen Thematisierungen von Zeitlichkeit bei Foto-, Film- und Videokünstler/innen seit den 199oer Jahren in den Blick genommen - ihre Manipulation der Bildbewegungen, die Konfrontation verschiedener Medien, die Stillstellung von Körpern im Bild, die Bearbeitung gefundenen Filmmaterials mittels filmischer Montage, den Möglichkeiten des Videorekorders und der Projektion im Ausstellungsraum. Dass die Darstellung von Räumen nicht von der Arbeit mit Zeit im bewegten Bild zu trennen ist, kann als selbstverständlich gelten: Die Zeit des Bildes muss sich im Raum zeigen, ihre Sichtbarmachung erfordert immer eine Ausdehnung in der Fläche, einen Grund und ein Bildfeld, in dem sich Kontraste manifestieren und Erscheinung ereignet. Die Verzeitlichung des Raums geht mit der Verräumlichung der Zeit einher, um nochmals Panofsky zu zitieren. Für viele Künstler und Künstlerinnen spielt die Inszenierung von Räumen eine ebenso große Rolle wie die Arbeit mit der Zeit. Dies betrifft einerseits die Form der Werke als kinematografische Installationen, die den Ausstellungsraum, die Rolle von Objekten und Bildern darin genauso neu definieren wie die Rolle der Betrachter/innen. Das Dispositiv des Kinos als Leitdifferenz zur Blackbox und zum White Cube dient der Orientierung wie auch der Abgrenzung, um den Ort der Installation zu definieren. Der Ausdruck >kinematografische Installation< hat sich mittlerweile als Sammelbegriff für alle Arten der Ein- und Mehrfachprojektion im Museums- oder Galeriekontext etabliert. ${ }^{1}$ Andererseits betrifft die Frage nach dem Raum und dem Ort des Bildes die Räume im Film, ihre bildliche Strukturierung mittels Perspektive, Kameraführung, Bewegung, Schnitt und Montage, Licht, etc. Die Orientierung der Zuschauer/innen, die Identifikation mit der Kameraposition und/oder einer Figur im Film unterstützt das mögliche Narrativ des Films.

Zeitgleich mit der seit den 199oern aufkommenden und bis heute anhaltenden Konjunktur filmischer Werke innerhalb des Kunstkontextes begann der kulturwissenschaftliche Trend, sich neu etablierende oder wieder aufflammende Diskurse als cultural turns unterschiedlicher Couleur zu

1 Vgl. u. a. die Publikation Frohne, Haberer, Kinematographische Räume und das Kapitel »Kinematographische Installation« in Rebentisch, Ästhetik der Installation. 
bezeichnen. Auffallend ist die Koinzidenz, dass simultan zur Hybridisierung des Bildes in der Kunst eine Diskussion über den Bildbegriff etabliert wurde, die bis heute anhält und als iconic turn eine Hinwendung zum Bild markiert. ${ }^{2}$ Mit dem spatial turn wurde jedoch ebenso die Wiederkehr des Raumes in den Kulturwissenschaften propagiert (in Abgrenzung zur Zeit, obwohl diese doch gerade in der Diskussion des Bildes eine große Rolle spielt), obgleich auch die Autoren des ersten Sammelbandes zum Thema Raum einen gewissen Selbstverstärkungseffekt durch verweisende Wiederholungen des angeblichen Paradigmenwechsels nicht ausschließen. ${ }^{3}$

Für die Betrachtung der folgenden Werke, die alle auf Film basieren - $16 \mathrm{~mm}$ und $35 \mathrm{~mm}$, zum Teil übertragen auf digitale Projektion -, bieten sich einige Unterscheidungen an: Auf der filmischen Ebene wird sich die Diskussion den fiktionalen, gestalteten Räumen widmen, und zwar mit Blick auf die Frage, wie bestimmte Orte mit filmischen Mitteln zeitlich und historisch definiert werden. Die Orte haben jedoch stets auch einen konkreten Bezug zur Wirklichkeit, es sind identifizierbare Plätze, deren architektonische und soziale Bedeutung thematisiert und in einen narrativen oder ästhetischen Zusammenhang gestellt wird. Die Werke von Mark Lewis und Tacita Dean zeugen weniger von einem philosophischen Raumbegriff, sondern offenbaren eine konkrete gesellschaftliche, historische, politische, persönliche - Bedeutung von Orten, für deren Erkundung und Darstellung die Zeit ein wesentliches Medium ist.

Verschiedene Ansätze, solche Orte und Räume näher zu bestimmen, können dabei hilfreich sein, so zum Beispiel die Unterscheidung zwischen >Räumen< und `Orten<, wie sie von Michel de Certeau als Teil einer Soziologie des Alltagslebens und Konsumverhaltens vorgenommen wurde:

Ein Ort ist die Ordnung (egal, welcher Art), nach der Elemente in Koexistenzbeziehungen aufgeteilt werden. [...] Ein Ort ist also eine momentane Konstellation von festen Punkten. [...] Ein Raum entsteht, wenn man Richtungsvektoren, Geschwindigkeitsgrößen und die Variabiliät der Zeit in Verbindung bringt. Der Raum ist ein Geflecht von beweglichen Elementen. Er ist gewissermaßen von der Gesamtheit der Bewegungen erfüllt, die sich in ihm entfalten. [...] Insgesamt ist der Raum ein Ort, mit dem man etwas macht. ${ }^{4}$

2 Maßgeblich wurde die Frage des Bildes durch Gottfried Boehms Sammelband Was ist ein Bild? (München 2001) vorangetrieben, ebenso durch die Beiträge von Hans Belting, Horst Bredekamp, Klaus Sachs-Hombach, W. T. Mitchell und James Elkins.

3 Jörg Döring, Tristan Thielmann, »Einleitung. Was lesen wir im Raume? Der Spatial Turn und das geheime Wissen der Geographen «, in: dies. (Hg.), Spatial Turn. Das Raumparadigma in den Kultur-und Sozialwissenschaften, Bielefeld 2008, S. 7-45.

4 Michel de Certeau, » Räumeく und 〉Orte««, in: ders., Kunst des Handelns, Berlin 1988, S. 217220, S. 217 f. 
De Certeau unterscheidet also zwischen einem »relationalen Raum der Erfahrung ${ } 5$ und einem stabilen, beispielsweise geometrischen Ordnungssystem der Lagebestimmung, den er »Ort« nennt. Er will mit seinem Raumbegriff eine Aufwertung der Alltagspraktiken erreichen, die durch ihre raumkonstituierende Wirkung die klare Machtordnung, wie sie Foucault in Überwachen und Strafen ${ }^{6}$ der Architektur zubilligte, aufbrechen kann. Seine Differenzierung hat für die Analyse künstlerischer Arbeiten den Vorteil, die Benennung des Ortes und der Bewegungen darin, welche einen Raum organisieren und strukturieren, voneinander $\mathrm{zu}$ trennen und ihre Interaktion so genauer beschreiben zu können. Lewis' und Deans Ortsstudien, die im Folgenden näher betrachtet werden sollen, zeichnen sich durch einige Parallelen, aber auch große Unterschiede aus. Beide nähern sich mit einem quasidokumentarischen Blick den Situationen, die sie filmen, sie kommen mit wenigen Schnitten beziehungsweise Kamerabewegungen aus, wodurch die Werke den Eindruck einer extremen Langsamkeit vermitteln, und sie setzen sich mit der Moderne als Paradigma auseinander. Ihre Behandlung realer Orte wirft Fragen auf nach der Unterscheidung von dokumentarischem und fiktionalem Gehalt des filmischen Bildes, nach der Rolle der Narration mittels Kamera- und Bildbewegung, nach Schnitt und Montage sowie nach der Bewertung der Historizität, wenn, wie bei Lewis, gezielt auch Mittel der Inszenierung eingesetzt werden.

\section{Heterotopia in Elephant and Castle. Vom Scheitern städtebaulicher Utopien}

»Ich mache gerne Filme über gewöhnliche Orte. [...]

Das sind Orte an denen wir die meiste Zeit verbringen.«

Mark Lewis ${ }^{7}$

Mark Lewis ist neben Stan Douglas und Jeff Wall einer der bekanntesten zeitgenössischen Künstler Kanadas. Er arbeitet seit vielen Jahren in London, kehrt aber immer wieder für einzelne Filme in seine Heimat zurück. In seinem Werk nimmt die großstädtische Kulisse Londons einen ebenso wichtigen Platz ein

5 Jörg Dünne, »Einleitung (Teil IV, Soziale Räume)«, in: ders., Stephan Günzel (Hg.), Raumtheorie. Grundlagentexte aus Philosophie und Kulturwissenschaften, Frankfurt am Main 2006, S. 289-303, S. 3 oo.

6 Michel Foucault, Surveiller et Punir. Naissance de la Prison, Paris 1975.

7 Mark Lewis in Mark Lewis - Nowhere Land, Regie: Reinhard Wulf, D 2o11, DigiBeta, 83' OF. 
wie die Landschaften Kanadas mit ihrer piktoralen Tradition. Innerhalb seines umfangreichen CEuvres, zu dem jährlich etliche Filme hinzukommen, findet sich ein Korpus von Werken, die problematischen städtebaulichen Situationen in seiner Londoner Wahlheimat gewidmet sind. In diesen Arbeiten über Wohnanlagen, Verkehrsknotenpunkte und Baustellen trifft eine spezifische Epoche britischer Städtebaupolitik der 1970er Jahre, die von der gescheiterten Utopie einer funktionalistischen modernen Architektur mit sozialistischen Stadtbauidealen zeugt, auf die Frage nach der passenden temporalen Form der Darstellung dieser historischen Ensembles. Lewis, der sich auch in theoretischen Texten mit der Frage nach der Bedeutung der Moderne und des Modernismus für die Gegenwartskunst auseinandersetzt, hat selbst als Anlass für diese Arbeiten das Ansinnen formuliert, daran zu erinnern, dass den Wohnungsbauprojekten ein idealistischer, utopischer Ansatz zugrunde liegt, nämlich für die Arbeiter/innen besseren Wohnraum zu schaffen. Die Bauprojekte scheiterten oftmals, weil es nicht gelang, die Realität und den Alltag der Bewohner/innen angemessen zu antizipieren. ${ }^{8}$ Christine Ross hat hervorgehoben, dass Lewis in seinen Filmen die Überbleibsel dieser modernen Bauweise nicht als ganz und gar vergangene, historische Ruinen behandelt, aber aufgrund der Tatsache, dass sie zum Zeitpunkt der Filme kurz vor dem Abriss standen, auch nicht als gegenwärtige Architekturen; vielmehr nähmen sie als »quasi-remnants« eine Position zwischen der Geschichte und der Gegenwart ein, eine Position, von der aus die Gegenwart als Modalität der Erfahrung in die Vergangenheit und in die Zukunft ausgedehnt wird: »They stretch the perceived event by connecting the now to the just-passed and the just-coming. ${ }^{9}{ }^{9}$ Ross vertritt damit einen ähnlichen Ansatz, wie er der vorliegenden Untersuchung zugrunde liegt. Er kann als Ausgangspunkt weiterer Überlegungen dienen: Wie genau gestaltet sich diese Korrelation der verschiedenen Zeitebenen im bewegten Bild? Welche Begriffe von 〉Ort $<$ und >Raum < vertritt Lewis? Welche Rolle spielen das Medium Film und die intermedialen Bezüge, vor allem zur Fotografie? Wie ist schließlich, auch im Vergleich zu Werken von Tacita Dean, das Verhältnis zur Realität in diesen doch explizit nicht dokumentarischen Kunstwerken zu definieren?

Der knapp vierminütige Film Tenement Yard (Abb. 22) besteht aus einer einzigen statischen Einstellung. Er wurde ebenso wie Children's Game (Heygate Estate) in Farbe und auf Super-35-mm gedreht und wird im Ausstellungsraum

8 Mark Lewis, Yilmaz Dziewior, »Gespräch mit Mark Lewis«, in: Yilmaz Dziewior (Hg.), Mark Lewis, Ausstellungskatalog Kunstverein in Hamburg, Hamburg 2005, Ostfildern-Ruit 2005, S. $47-56$, S. 5 .

9 Ross, The Past is the Present; It's the Future, Too, S. 140. 

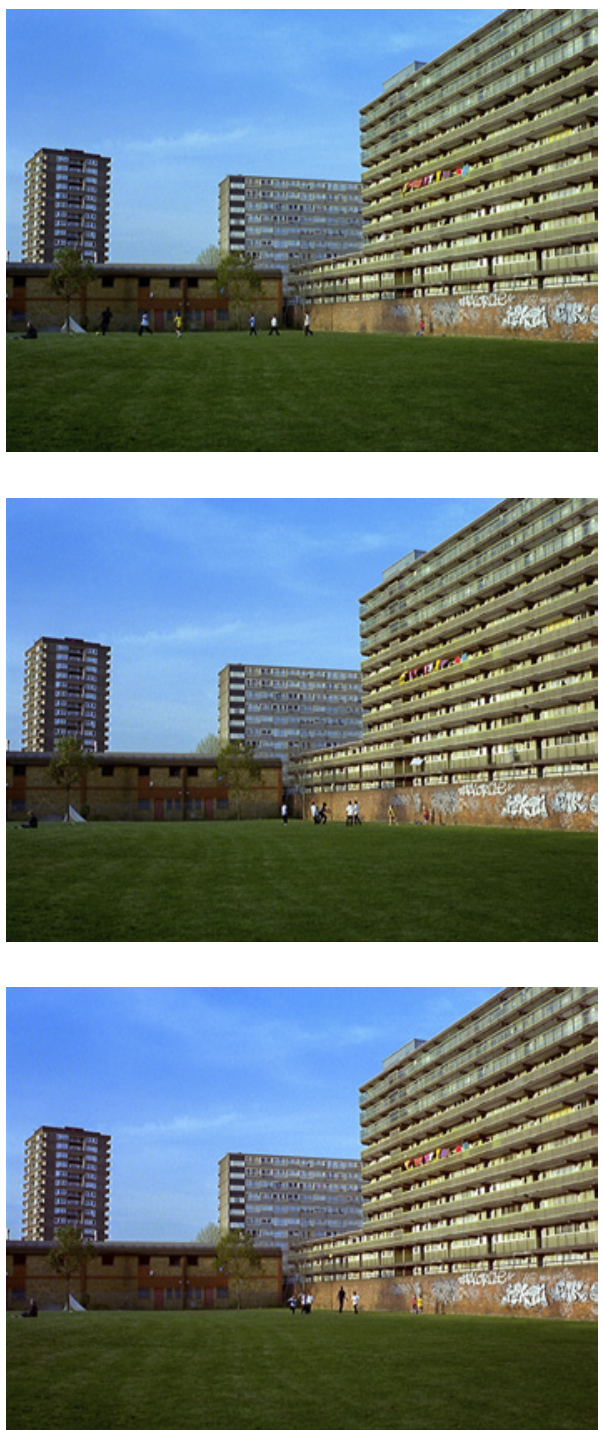

Abb. 22

Mark Lewis, Tenement Yard

(Heygate Estate), 2002 
digital projiziert. Die Einstellung zeigt im Vordergrund eine dunkelgrüne Grasfläche, die im Mittelgrund in einer Ecke mündet. Die linke sichtbare Seite der Grasfläche ist durch ein flaches, gelb-rotes Backsteingebäude mit einigen Fenstern begrenzt, davor stehen zwei schmale Bäume. Hinter dem Flachbau erhebt sich ein backsteinverkleidetes Hochhaus in den Himmel. Rechts von der Grasfläche befindet sich eine mannshohe, mit Graffiti verzierte Steinmauer, welche sich von vorn bis hinter den Flachbau durch den Bildraum zieht. Wo Mauer und Gebäude sich treffen, liegt auf dem goldenen Schnitt der Mittelpunkt des Bildes. Hinter der Steinmauer ragt in schräger Unteransicht ein hoher Wohnblock ins Bild, dessen horizontale Gliederung den nach außen verlagerten Zugängen zu den Wohnungen geschuldet ist. Im Hintergrund, halb von diesem verdeckt, schließt ein weiterer Wohnblock das Ensemble ab. Zwar ist der Blick in den Hinterhof starr, und die Architektur bildet einen betonierten, fixen Rahmen, dennoch wirkt die Szenerie erstaunlich bewegt. Auf der Rasenfläche spielen Kinder Fußball, in einem der oberen Stockwerke des Wohnblocks flattert bunte Wäsche zum Trocknen im Wind. Neben dem die Wahrnehmung auf sich ziehenden Fußballspiel, das die Horizontale der Wiese betont, sind zwei Objekte weitere Hauptakteure der sich im Bild ereignenden Handlungen: zwei weiße Matratzen, welche von mehreren Personen über die unterste sichtbare Etage des rechten Gebäudes getragen werden. Sie gelangen von rechts ins Bild und verschwinden nach einiger Zeit in einem Aufgang, um aus diesem gegen Ende des Films, immer noch mit Matratzen in den Händen, wieder herauszutreten und den Balkon bis zum rechten Bildrand zurückzulaufen.

Children's Games (Heygate Estate) (Abb. 23) hat ebenfalls nur eine einzige Einstellung, diese ist jedoch ungleich bewegter. Dieser 7:29 Minuten lange Film besteht aus einer durchgehenden Kamerafahrt, die von einer Steadicam aufgenommen wurde, welche sich auf einer Art handgeschobener Rikscha befand. Das Gefährt bewegt sich einen Fußgängerweg entlang, der sich über zahlreiche Brücken und Kurven durch die in den frühen 196oer Jahren entstandenen Wohnblocks des Heygate Estate in Londons Viertel Elephant and Castle schlängelt. Ohne Schnitte passiert die Kamerafahrt zahlreiche Kreuzungen, Plätze, Abzweigungen und Unterführungen, überwindet Höhenunterschiede und endet schließlich an einer befahrenen Straße. Während des Films begegnet die Kamera keinen Fußgängern oder Autos, es bevölkern jedoch zahlreiche spielende und rennende Kinder und Jugendliche die Randbereiche des Bildrahmens. Diese spielen Ball, lassen Drachen steigen, fahren Fahrrad oder erfüllen auf andere Art und Weise die monumentale, modernistische Architektur mit Leben. 

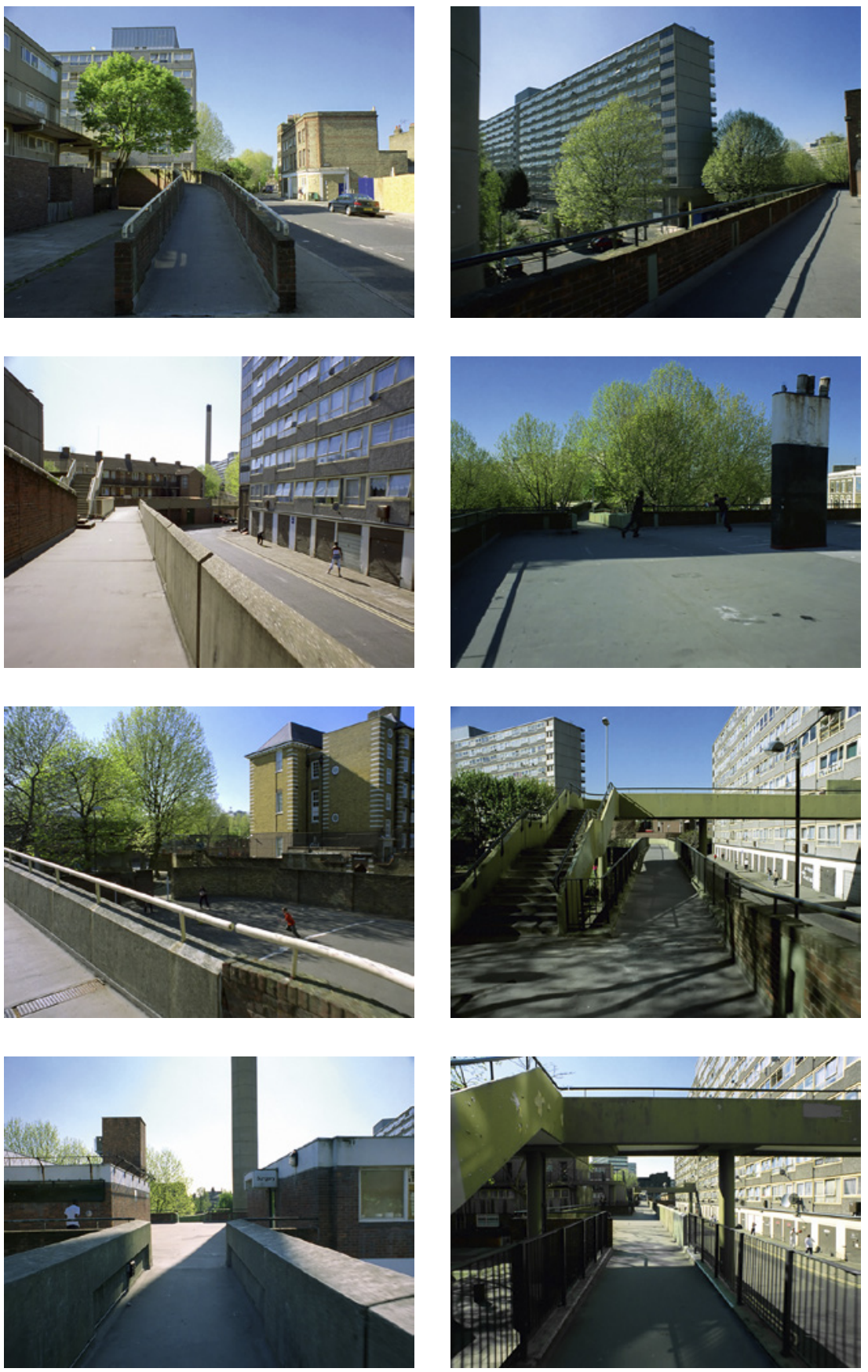

Abb. 23

Mark Lewis, Children's Games (Heygate Estate), 2002 
Das in beiden Filmen die Kulisse stellende Areal Heygate Estate war ein zentrales Projekt des sozialen Wohnungsbaus in London und stellte Wohnraum für 3000 Menschen. Es wurde in den 196oer Jahren geplant und 1974 in Londons Süden vollendet. Der Abriss erfolgte zwischen 2011 und 2014. An seiner Stelle, in relativ zentraler Lage Londons, werden neue Wohnhäuser und Anlagen errichtet, zum Teil durch die öffentliche Hand, zum Teil privat. Die Umstrukturierung und Neubebauung des Viertels Elephant and Castle hat bei den Bewohnern des Heygate Estate massive Proteste hervorgerufen und ist auch finanziell umstritten, da solch großangelegte Wohnungsbauprojekte zur Amortisierung einer längeren Nutzungsdauer bedürfen. Letztlich konnten sich die Befürworter des Abrisses mit Verweis auf soziale Probleme und Kriminalität in den Wohnblocks durchsetzen. Die Architektur des Heygate Estate war vom normierten, funktionalen Design des sozialen Wohnungsbaus der 1970er Jahre geprägt. Beton als vorherrschendes Material dominierte das Erscheinungsbild der zwei langgezogenen Wohnblöcke mit eintöniger Fensterfront. Die zahlreichen Fenster erlaubten sehr helle, lichte Wohnungen, die im Gegensatz zur traditionellen Architektur englischer Arbeitersiedlungen standen; durch die hochgelagerten Fußgängerwege, die Lewis in seinem Film erkundet, sollten die Passanten vom Lärm und Schmutz der Straßen ferngehalten werden. Grünflächen zwischen den Blöcken dienten der Auflockerung und Erholung. In den Reaktionen der Presse auf den Abriss der Gebäude wird deutlich, dass die Einwohnerstruktur des Heygate Estate sehr gemischt war und die Bewohner bis zuletzt die Gründe für die Neustrukturierung nicht teilten, sondern im Gegenteil die Probleme des Viertels als nicht gravierend wahrnahmen. Gerade im hochpreisigen London wurde der Wegfall bezahlbarer Wohnungen in Zentrumsnähe sehr bedauert und die Profitgier der Investoren angeprangert. ${ }^{10}$ Die ursprünglichen Bauten verfolgten den idealstädtischen Ansatz einer Gartenstadt, in welcher der belastende Verkehr reduziert werden und im Gegenteil die Position der Fußgänger/innen gestärkt werden sollte. Moderne, große Wohnungen mit zeitgemäßer Sanitärausstattung, eine funktionierende Infrastruktur und eine gute Anbindung an den öffentlichen Nahverkehr machten solche Areale, wie beispielsweise auch das Märkische Viertel in Westberlin und die Bebauung der Fischerinsel im ehemaligen Ostberlin, zu begehrten Wohnorten im Gegensatz zu verfallenden, schlecht instandgehaltenen Altbauten. Wurden in Großbritannien in der Nachkriegszeit bis ca. 1975 angesichts des Wohnungsmangels infolge der Kriegsschäden Bauprojekte durch die öffentliche Hand massiv vorangetrieben, änderte sich

10 Vgl. beispielsweise Stephen Moss, »The death of a housing ideal«, in: The Guardian, 4.3.2011, https://www.theguardian.com/society/2011/mar/o4/death-housing-ideal [26.4.2020]. 
unter der konservativen Thatcher-Regierung die Wohnungsbaupolitik. Seit den 199oer Jahren wird die Privatisierung von Wohneigentum gefördert und das Council Housing reduziert. Durch die Housing Acts von 1980 und 1988 wurde es vor allem einkommensstärkeren Haushalten ermöglicht, Wohnungen günstig zu kaufen, wodurch sich die Bewohnerstruktur der kommunalen Mietshäuser stark wandelte und die Konzentration einkommensschwacher Haushalte gefördert wurde. Heute gelten viele dieser Siedlungen als Problembezirke, und das Wohnideal hat sich von monumentalen Anlagen weg und hin zu kleinteiligen Quartierstrukturen sanierter Altbauten in privater Hand verschoben. Heygate Estate kann somit als Beispiel für die idealistischen Ziele des öffentlichen Wohnungsbaus in den 197oer Jahren dienen, der bezahlbaren Wohnraum für alle schaffen sollte, jedoch durch den politischen Kurswechsel und die gleichzeitige Vernachlässigung der bestehenden Infrastruktur ein vorzeitiges Ende fand.

Mark Lewis' Werke thematisieren die genannten sozialpolitischen und gesellschaftlichen Umstände des Heygate Estate nicht direkt, diese bilden jedoch den historischen Hintergrund der beiden Filme. Lewis' Augenmerk ist auf die räumlichen Bedingungen des Wohnens dort gerichtet, und hier besonders auf die Situation der Kinder. Er idealisiert die Trostlosigkeit der Architektur nicht, die in ihrer Monumentalität den einzelnen Menschen zur winzigen Staffagefigur werden lässt, sondern er eröffnet durch die sorgfältige Inszenierung des kindlichen Spiels einen Raum für die Erinnerung an das utopische Potenzial der Anlage. Die beiden Filme unterscheiden sich stark in ihrer bildlichen und dynamischen Erfassung des Raumes. In Tenement Yard bildet die statische Architektur zusammen mit der statischen Kameraeinstellung den Rahmen, in dem sich die sich nur in kleinen Details zeigende Bewegung entfaltet. Die rechte Bildhälfte ist von der diagonalen Ansicht des Wohnblocks dominiert, in dessen Verlängerung sich die beiden anderen Häuser befinden. Sie nehmen den Großteil des Bildfeldes ein. Das eintönige Rasenstück und der blaue, konturlose Himmel bilden nur einen schwachen Kontrast zueinander. Der Blick des Betrachters / der Betrachterin heftet sich an bewegte Details: die rennenden Kinder, welche den Ball hochschießen, flatternde Wäsche im Wind auf einem Balkon des Wohnblocks, schließlich die weiße Matratze, die über die unterschiedlichen Stockwerke getragen wird. Wird der >Ort< des Bildes durch die perspektivischen starren Linien der Architektur vorgegeben, in die sich auch die Natur als undefiniertes Areal einzufügen hat, so schaffen die Bewegungen den $>$ Raum $<$, in dem sich städtisches Leben ereignet. Genau inszenierte Bewegungslinien wie die der Matratze sind mit der zufälligen, sich aus dem freien Spiel der Kinder ergebenden Ball- und Laufbewegung im unteren Bildfeld kombiniert. Sie brechen die geometrische 
Struktur der Architektur immer wieder auf und lassen die Zufälligkeit der Bewegung einen eigenen Handlungsraum definieren. Children's Games (Heygate Estate) ist fast doppelt so lang wie Tenement Yard, hat jedoch durch Beginn und Ende der Kamerafahrt eine den Film durchgehend bestimmende Aktion. Wird bei ersterem Film die monumentale Architektur mit ihrer Betonung der Vertikalen den Versuchen der Rückeroberung des Raums durch soziales Handeln radikal entgegengestellt - ohne den Konflikt zugunsten einer Seite aufzulösen -, so wird hier der Ansatz verfolgt, sich kraft der buchstäblichen >Erfahrung< des Areals dessen Ordnung anzueignen, den Ort durch Bewegung zu erfassen und zum Raum werden zu lassen. Die Kamerafahrt - eine Reminiszenz an die verbreiteten phantom rides der frühen Kinematografie, wie sie im experimentellen Film immer wieder aufgegriffen werden,$-{ }^{11}$ erzeugt eine spezielle Dynamisierung des Raumes, welche die fixierten Betrachter/ innen die Umwelt als vorbeifliegende >Ansicht< erleben lässt. Die an den Bildrändern sporadisch auftauchenden Kinder bewegen sich ungeordnet und teils gegenläufig zu der durch die Wegführung vorgegebenen Kamerabewegung und haben auch hier wieder die Funktion, die Aneignung des Ortes mittels des Spiels zu symbolisieren.

Michel de Certeau grenzt sich in seiner Kunst des Handelns bewusst von Foucaults These ab, dass die Disziplinierung der menschlichen Praktiken durch die machtbündelnden Institutionen alle Lebensbereiche erfasst habe, und plädiert dafür, dem mittels Alltagspraktiken entgegenzuwirken: Dem »Raummodell der visuellen Kontrolle [...] stellt er die Raumpraxis >von unten< entgegen, die er mit der Tätigkeit des Laufens durch die Stadt identifiziert «. ${ }^{12}$ Lewis' Betonung des Spiels und die Kontrastierung der normierten Architektur mit dem aleatorischen Bewegungspotenzial der Kinder kann als Aufnahme dieses Gedankens gedeutet werden. In Children's Games (Heygate Estate) wählt er mit der Fußgängertrasse, die einzig der Passage zwischen den einzelnen Gebäuden dient, einen Ort aus, der in die Definition von Marc Augés

11 Zum Beispiel Ernie Gehrs Found-Footage-Film EUREKA (1974) oder Stan Douglas' Installation Overture (1986). Siehe dazu auch Christa Blümlinger, »Lumière, der Zug und die Avantgarde«, in: Malte Hagener (Hg.), Die Spur durch den Spiegel. Der Film in der Kultur der Moderne, Berlin 2004, S. 27-41.

12 Dünne, »Einleitung «, S. 299. Certeau schreibt selbst: »Wenn es richtig ist, daß das Raster der >Überwachung « sich überall ausweitet und verschärft, dann ist es um so notwendiger, zu untersuchen, wie es einer ganzen Gesellschaft gelingt, sich nicht darauf reduzieren zu lassen: welche populären (und auch >verschwindend kleine $<$ ) Praktiken spielen mit den Mechanismen der Disziplinierung und passen sich ihnen nur an, um sie gegen sich selber zu wenden; und welche Handlungsweisen bilden schließlich auf Seiten der Konsumenten (oder >Beherrschten<?) ein Gegengewicht zu den stummen Prozeduren, die die Bildung der soziopolitischen Ordnung organisieren? (Certeau, Kunst des Handelns, S. 16.) 
>Nicht-Orte< fällt. Augé konkretisiert den Begriff, den bereits De Certeau verwendet, ${ }^{13}$ für Durchgangsorte wie Bahnhöfe, Flughäfen und Einkaufzentren, die allein für bestimmte Zwecke, zum Beispiel den Transit oder den Handel, geschaffen wurden: »Wie man leicht erkennt, bezeichnen wir mit dem Ausdruck Nicht-Ort zwei verschiedene, jedoch einander ergänzende Realitäten: Räume, die in Bezug auf bestimmte Zwecke (Verkehr, Transit, Handel, Freizeit) konstituiert sind, und die Beziehung, die das Individuum zu diesen Räumen unterhält. «14 Die Nicht-Orte verändern für die Zeit des Aufenthalts die Bestimmungen des Individuums, sie haben ihre eigenen Verhaltens- und Kommunikationsregeln. In der Realität existieren sie jedoch selten in Reinform, denn Orte und Nicht-Orte durchdringen sich: »Die Möglichkeit des Nicht-Ortes ist an jedem beliebigen Ort gegeben $\ll,{ }^{15}$ gleichzeitig kann er jederzeit wieder verlassen werden.

Ähnlich konzipiert wie die Idee des Nicht-Ortes, jedoch weniger anthropologisch ist Foucaults Begriff der >Heterotopie<, der im Anschluss an zwei Radiovorträge 1966 und einen kurzen Aufsatz, der 1967 geschrieben, aber erst 1984 veröffentlicht wurde, eine erstaunliche Karriere in den Kulturwissenschaften und der Architekturtheorie gemacht hat. ${ }^{16} \mathrm{In}$ »Von anderen Räumen « charakterisiert Foucault das moderne Raumdenken als relational, der Raum biete sich in Form von »Relationen der Lage $«{ }^{17}$ dar. Was er als Heterotopie beschreibt, ist ein Raum, dem »die merkwürdige Eigenschaft zukommt, in Beziehung mit allen anderen Orten zu stehen «, ebenso, wie es bei seinem Gegenstück, der Utopie, der Fall ist. Utopien sind nach Foucaults Definition gleichzeitig Orte und Bilder: Sie sind für ihn »Orte ohne realen Ort«, »zutiefst irreale Räume«, die sich aber trotzdem in einem Analogieverhältnis zum wirklichen Raum der Gesellschaft befinden und so gesehen ein ideales Bild oder ein Gegenbild derselben darstellen. ${ }^{18}$ Die Heterotopie ist als auch zeitlich divergenter Gegenort (Heterochronie) zur irrealen, weil nicht verwirklichbaren Utopie konzipiert und unterscheidet sich zugleich von herkömmlichen Orten dadurch, dass sie als quasi realisierte - weil an realem Ort und in realer

13 Auch Robert Smithson spricht schon in den 196oer Jahren von sogenannten >nonsites $<$. Er verwendet den Ausdruck jedoch für Werke in Galerien oder Ausstellungen, im Gegensatz zu seinen ssites<, die Land-Art-Projekte, welche an bestimmten Orten realisiert werden.

14 Marc Augé, Nicht-Orte, 2. Auflage, München 2011, S. 96.

15 Ebd., S. 107.

16 Siehe Michel Foucault, Die Heterotopien. Der utopische Körper, Frankfurt am Main 2005; Michel Foucault, »Von anderen Räumen«, in: ders., Schriften in vier Bänden. Dits et Ecrits, Bd. 4, hg. v. Daniel Defert, Francois Ewald, Frankfurt am Main 2005, S. 931-942.

17 Vgl. Foucault, »Von anderen Räumen«, S. 933.

18 Vgl. ebd., S. 935 . 
Zeit befindliche - Utopie mehrere Funktionen und Orte zusammenbringt (wie beispielsweise das Kino, das Theater, den Garten, das Schiff, das Fest etc.). Foucaults Heterotopien haben sehr konkrete, auch zeitlich begrenzte Funktionen, in denen die Regeln des Alltagsraums außer Kraft gesetzt werden, wie bei der Bewältigung von Krisen oder dem Aufenthalt in Sanatorien oder Gefängnissen, wo Abweichungen von der Verhaltensnorm behandelt werden.

Das Heygate Estate erscheint in Mark Lewis' filmischen Darstellungen, welche die soziopolitische Realität und die architektonische Irrealität miteinander konfrontieren, als ein Areal, das eine Heterotopie und zugleich ein Nicht-Ort ist; als real gebaute Utopie, in deren abgeschlossenem Raum der Wohnungsmisere im Großbritannien der 1970er Jahre begegnet werden sollte, die aber letztlich dieses Ideal nur zeitlich begrenzt erfüllen konnte. Die Hoffnung, durch das freie Spiel der Kinder die zementierte Ordnung eines Lebens- und Freizeitraumes für die arbeitenden Massen aufbrechen zu können, kann nur zeitlich begrenzt eingelöst werden - auf Dauer ist dieses Projekt in der Realität zum Scheitern verurteilt. Ob dies auch an solchen NichtOrten wie den Fußgängertrassen liegt, die ausschließlich der Passage, aber nicht der Begegnung und Öffnung durch die Bewohner/innen dienen, bleibt offen. ${ }^{19}$ Mit der Kamerafahrt suggeriert Lewis, dass sie immerhin der vielbefahrenen Straße außerhalb des Areals Heygate Estate vorzuziehen sind.

Die Korrelation von zeitlicher filmischer Erfahrung und der Darstellung von Geschichte ist in den zwei kurzen Filmen augenfällig und stellvertretend für Lewis' weiteres filmisches Werk: Die statische und die von außen bewegte Kamera weisen zwei Zugänge zum filmischen Bild auf, die auf die klassische Montage und Handlungsorientierung verzichten und an die Techniken der Frühzeit des Kinos anschließen. Für die These der Verknüpfung von sogenannten Nicht-Orten und ihren Funktionen als Heterotopien spricht das anhaltende Interesse Lewis' für die städtebaulichen Randbereiche, die der Passage dienen und durch ihre besondere zeitliche Abgeschlossenheit und Organisation auffallen: Flughäfen, Straßenkreuzungen, Museen oder Aufzüge

19 Nach Augé wären diese Trassen als reine Durchgangsräume zu werten. Certeau hingegen wertschätzt das Zu-Fuß-Gehen als performativen Akt, da die Fußgänger/innen mittels ihrer Bewegung den urbanen Raum allererst erzeugen: »Der Akt des Gehens ist für das urbane System das, was die Äußerung (der Sprechakt) für die Sprache oder für formulierte Aussagen ist. [...] Die Spiele der Schritte sind Gestaltungen von Räumen. Sie weben die Grundstruktur von Orten. In diesem Sinne erzeugt die Motorik der Fußgänger eines jener realen Systeme, deren Existenz eigentlich den Stadtkern ausmacht, die aber keinen Materialisierungspunkt haben. Sie können nicht lokalisiert werden, denn sie schaffen erst den Raum.«(Certeau, Kunst des Handelns, S. 188 f.) 
und Rolltreppen. Sein filmisches Vokabular wendet auf die Orte präzise, teils im Titel genannte Bewegungen der Kamera oder andere filmische Mittel, die bereits im frühen Film eingesetzt wurden (Schärfeverlagerung, Rückprojektion, Verlangsamung, Rückwärtslauf des Films), an. Seine Filme, die einerseits mit dokumentarischem Gestus reale Orte und ihre historische Bedeutung erfassen und andererseits durch die sorgfältige Inszenierung minimaler Handlungen und Geschichten den imaginären Raum des Kinos öffnen, fungieren mitunter selbst als Heterotopien, als die Realität spiegelnde, ihr utopisches Potenzial sichtbar machende bewegte Bildräume. Seinem Interesse an der Frage, »wie sich verschiedene Zeitarten gleichzeitig im selben Werk beschreiben lassen «, 20 tragen die Werke Rechnung, indem sie das zeitliche Bewegungspotenzial eines konkreten historischen Raumes offenzulegen versuchen, also auch einzelne lokale Bewegungen innerhalb der Dauer einer Einstellung synchronisieren.

\section{>Andere Räume`im Werk von Tacita Dean}

Die Interrelationen von verschiedenen Zeitebenen und spezifischen Orten, die zugleich eine fiktionale und dokumentarische Dimension zeigen, spielen im Werk von Tacita Dean von Beginn an eine besondere Rolle. Ähnlich wie Lewis interessiert sie sich für die Orte, die als >andere Räume`spezielle Funktionen einnehmen und vielfach auch wie Heterotopien als reale Spiegelungen utopischer Räume fungieren. Das Schiff, von Foucault als Heterotopie par excellence bezeichnet, ist ein vorherrschendes Thema ihrer Zeichnungen. Die Filme der letzten Jahre zeigen die Überbleibsel politischer Utopien als Ruinen oder, wie bei Fernsehturm, als abgeschlossenen Raum mit einer eigenen zeitlichen Ordnung, sie zeigen futuristische Gebäude, Ateliers, Studios, Museen, ein Kloster und einen Garten. Anders als Lewis nimmt sie jedoch nicht konkret auf das formale Vokabular der Filmgeschichte Bezug und arbeitet auch nicht mit Schauspielern, welche die möglichen alltäglichen Praktiken vor Ort nachspielen, sondern versucht, die dem jeweiligen Ort eigene Dynamik einzufangen. Ihre Filme bedienen sich nicht narrativer Mittel, um die Bildzeit (nach Theissing das Zusammenspiel der zeitlichen Dimensionen des Dargestellten und der temporalen Qualität des Bildes) zu vermitteln, sondern, wie aus den bereits betrachteten Arbeiten deutlich wurde, der Dauer statischer

$20 \quad$ Lewis, Dziewior, »Gespräch mit Mark Lewis«, S. 51. Zur Kombination verschiedener Zeitlichkeiten bei Lewis in Bezug auf die Frage nach einer Globalzeit siehe auch den Aufsatz von Élie During, »Cinematography and the Extended ^Nowく. From Bergson to Video Art«, in: Rathgeber, Steinmüller, BildBewegungen/ImageMovements, S. 256-279. 
Einstellungen und des Blickes auf Details. Orte sind bei Dean vor allem die imaginären Orte, die sich auf eine Fiktion - einen Mythos, eine Geschichte, eine politische Utopie - beziehen und mittels ihres dokumentarischen Stils präsentiert werden.

Im Weiteren sollen einige ihrer Werke anhand der Hypothese analysiert werden, dass sie die Orte vor allem über die Heterochronien, die zeitlichen Brüche, ins Bild setzt und so als Schnittstellen zwischen verschiedenen zeitlichen Ordnungen definiert.

»Eine Heterotopie beginnt erst dann ganz voll zu funktionieren, wenn die Menschen einen absoluten Bruch mit der traditionellen Zeit vollzogen haben. ${ }^{21}$ Foucault beschreibt die komplexe Struktur der Heterotopien anhand ihrer Räumlichkeit, also der Fähigkeit, mehrere Räume miteinander zu vereinen, aber auch anhand ihrer widersprüchlichen Zeitlichkeit: Sie weisen - wie die Utopien auch - einen konkreten Bezug zur Wirklichkeit auf, um sich von dieser durch die Reflexion ihrer Zeitstruktur abzugrenzen. In Bezug auf die Zeit heißt dies, als Heterotopien/-chronien gelten auf der einen Seite die Orte, die sich der >Anhäufung von Zeit< verschrieben haben, wie die neuzeitlichen Museen und Bibliotheken mit ihren umfassenden Sammlungen (»Der Gedanke, alles zu sammeln, gleichsam ein allgemeines Archiv aufzubauen [...], einen Ort für alle Zeiten zu schaffen, der selbst außerhalb der Zeit steht $\aleph^{22}$ ), auf der anderen Seite die Orte, die »mit den flüchtigsten, vergänglichsten, prekärsten Aspekten der Zeit verbunden sind, und zwar in Gestalt des Festes «. ${ }^{23}$ Hierbei geht es um ausschließlich temporär genutzte Orte, die einige Wochen im Jahr Jahrmärkte, Messen etc. beherbergen.

Es ist lohnenswert, hierfür noch einmal zu Deans Beschäftigung mit Merce Cunningham zurückzukehren. In Kapitel 3 wurden Cunninghams Performance und Deans Filmprojekt STILLNESS beschrieben, die in einem kleinen Trainingsraum der Merce Cunningham Studios in New York aufgezeichnet wurden. Wie für ein Tanzstudio üblich, befindet sich auf einer Seite ein großer Spiegel, in dem die Tänzerinnen und Tänzer ihre Bewegungen und Haltungen kontrollieren können. Den Spiegel nennt Foucault an sich schon ein Beispiel für einen heterotopischen Ort, da er die Zeitlichkeit des realen mit dem des imaginären Ortes der Spiegelung verschränkt. Doch auch das Studio selbst weist Funktionen auf, die den beschriebenen Grundsätzen der sogenannten sanderen Räume< Foucaults entsprechen: So, wie Heterotopien ein System der Öffnung und Abschließung voraussetzen, hat auch das Studio

$\begin{array}{ll}21 & \text { Foucault, »Von anderen Räumen«, S. } 939 . \\ 22 & \text { Ebd. } \\ 23 & \text { Ebd. }\end{array}$ 
eine strenge Zugangsbeschränkung und einen sehr spezifischen Zweck, es ist ein Ort der körperlichen Arbeit und der Ausbildung, in dem die Tänzer/innen nach bestimmten, strikt festgelegten Mustern die Tanztechnik Cunninghams erlernen, die wiederum auf andere Räume, die Bühnen, übertragen werden. Deans Filme erfassen diesen Ort mit seiner Verschränkung der heruntergekommenen Wände, des geschundenen Holzbodens und des Spiegels, an dem die Spuren tänzerischer Bewegungen als Abdrücke sichtbar sind, und verlegt ihn in die Zweidimensionalität des projizierten Leinwandbildes; aus den sechs Leinwänden wird in der Installation jedoch erneut ein begehbarer Ort, der zeitgleich verschiedene Einblicke in das Studio, auf Cunningham und sein Spiegelbild (und das Spiegelbild des gefilmten Raumes) erlaubt.

In dem zweiten Film, den Dean mit Cunningham drehte, gehen ebenfalls unterschiedliche Räume an einem Ort ineinander über und bilden ein heterotopisches Gefüge. Craneway Event (Abb. 24-26) ist die Dokumentation der dreitägigen Vorbereitung einer Tanzaufführung der Cunningham Company, die der Choreograf in der Kranbahnhalle eines ehemaligen Ford-Montagewerks in Richmond, Kalifornien, 2009 leitete.

Dean begleitete die Trainings in der von Albert Kahn gestalteten Halle mit Blick über den Frachthafen San Franciscos. Die 1931 erbaute, lichtdurchflutete Halle wurde zunächst für die militärische Fahrzeugproduktion genutzt, später auch als Filmset und, nach starken Erdbebenschäden 1989, erst ab 2004 renoviert und als Veranstaltungsort etabliert. Für die Aufführungen trainierte die Cunningham Company mehrere Tage vor Ort auf großen Holzpodesten, was von Dean mit der Kamera dokumentiert und zu einem 16-mm-Farbfilm von 108 Minuten Länge geschnitten wurde. Dieser wird im Ausstellungskontext in einer kinoähnlichen Situation, das heißt in einem abgeschlossenen, verdunkelten Raum mit Sitzgelegenheiten gezeigt. ${ }^{24}$ Die lichte, moderne Industriearchitektur wird von den umlaufenden bodentiefen Fenstern erhellt, die es ermöglichen sollten, das Tageslicht optimal für die Produktion auszunutzen; für die Tänzer/innen bedeutet dies, dass sich ihre Silhouetten im Film vor den stählernen Gitternetzen der Fenster und dem Himmel scherenschnittartig abzeichnen; der weite, offene Raum verschmilzt mit dem Außenraum der Hafenszenerie und der fernen bergigen Landschaft der San Francisco Bay Area, das Wasser reflektiert das Licht der langsam sinkenden Sonne, und die

24 Ursprünglich war das Ziel, beim fertigen Film den Eindruck eines einzelnen Trainings zu erwecken, weshalb Dean die Tänzer und Tänzerinnen gebeten hatte, jeden Tag die gleiche Kleidung anzuziehen. Aufgrund eines Kleidungswechsels von Merce Cunningham und unterschiedlichen Lichtsituationen an den drei Tagen musste dieses Vorhaben jedoch aufgegeben werden. 

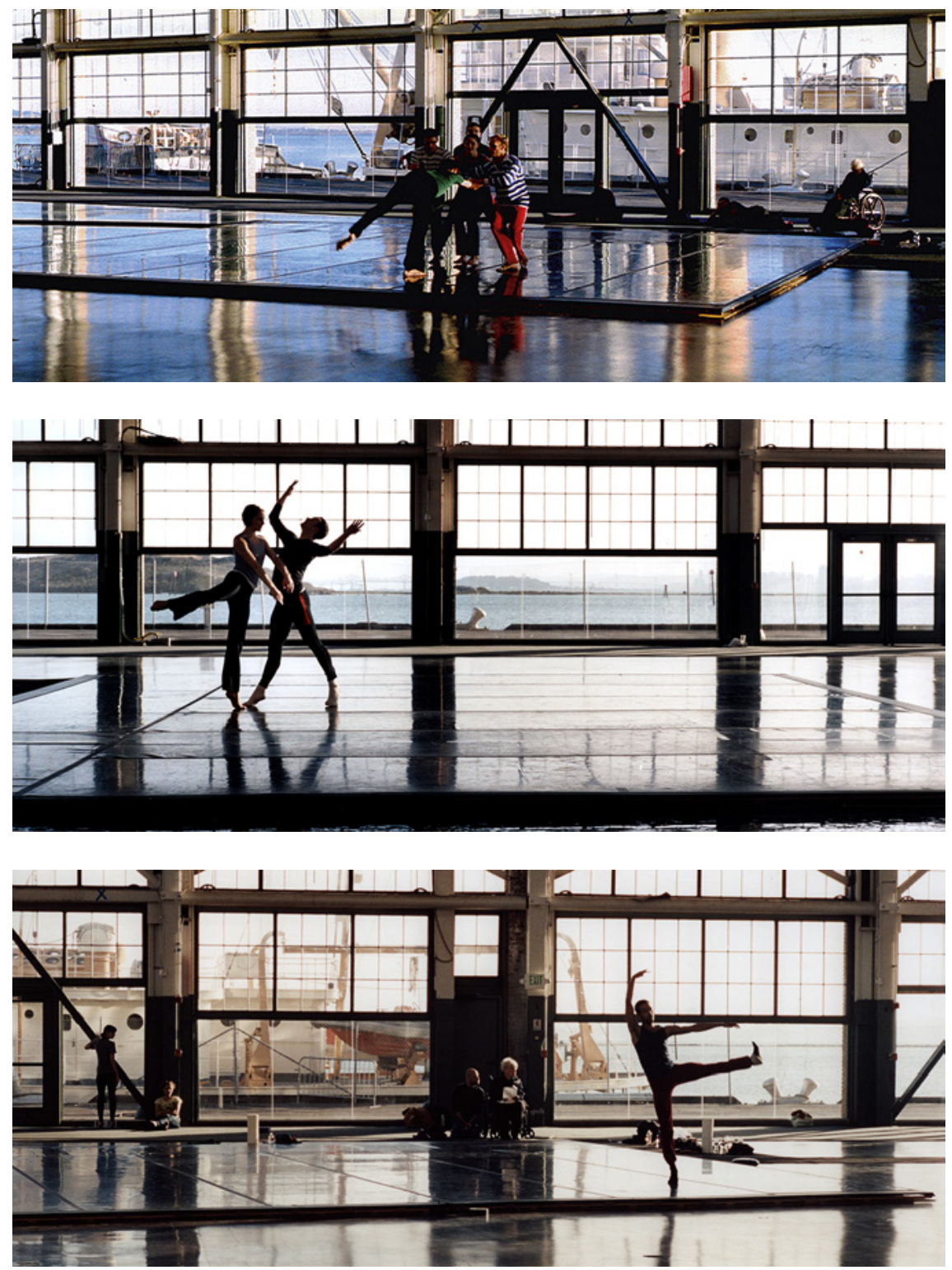

Abb. 24-26 Tacita Dean, Craneway Event, 20o9, Filmstills 
Farben des Himmels werden wiederum vom spiegelnden Boden der Halle reflektiert, so dass Innen- und Außenraum über das Licht- und Schattenspiel ineinander überzugehen scheinen. Der anamorphotisch aufgenommene Film erfasst die Breite der Halle, es dominieren Einstellungen in der Totalen, welche die Figuren der Tänzer/innen beim Aufwärmen und Trainieren im Ganzen erfassen. Cunningham hatte drei Bühnenpodeste nebeneinander installieren lassen, auf denen jeweils einzelne Stücke aus seinem Repertoire getanzt wurden. Jeder Tänzer und jede Tänzerin zählte dabei selbst seinen/ ihren Takt, eine zeitliche Abstimmung zwischen den Bühnen erfolgte nicht, einzig Cunningham koordinierte in den Besprechungen die zu tanzenden Ausschnitte in ihrer räumlichen Orientierung. So hatte jede Bühne und darauf jeder Tänzer und jede Tänzerin eine eigene, raumzeitliche Ordnung, welche aber natürlich auf der Trainingstechnik Cunninghams und seinem speziellen Umgang mit Zeit und Raum in den Stücken basierte.

Visuell leitend für die Komposition der Bilder ist die Verschmelzung von Innen- und Außenraum - durch die Spiegelung setzt sich die schillernde Wasseroberfläche auf den Podesten fort, vor den tiefen Fenstern ziehen gemächlich Fischkutter, Fracht- und Segelschiffe vorbei, man sieht schnelle Motorboote und zahlreiche Seevögel. Zu Beginn des Films beobachtet die Kamera minutenlang einen Pelikan, der sich draußen auf einem Poller niedergelassen hat, und einmal verirrt sich eine Taube in die Halle, die auf dem spiegelnden Boden umherspaziert. Cunningham selbst sitzt während der gesamten Zeit aufmerksam in seinem Rollstuhl und dirigiert die Proben, bespricht sich mit seinen Tänzer/innen und kommentiert die beeindruckenden Ausblicke. Wie üblich wird nicht zu Musik trainiert, so dass die Geräuschkulisse des Hafens nur von den Geräuschen der Bewegungen der Tänzer/innen selbst durchbrochen werden, ihren Schritten und Sprüngen auf den Podesten, dem Atmen und Keuchen, schließlich den Gesprächsfetzen bei den Besprechungen.

»Light, like the sea, travels in waves. Such metaphors are central to Tacita Dean's work «, 25 schreibt Peter Wollen und benennt somit die zwei wichtigsten Motive in Deans Arbeit. Wie in einigen anderen Filmen spielt das Licht im Zusammenspiel mit dem Wasser eine wesentliche Rolle in Craneway Event, denn beide Elemente symbolisieren in ihrer ständigen Bewegung und Flüchtigkeit den Fluss der Zeit: $\gg[D]$ ie Immaterialität der Zeit findet [...] ihre Entsprechung in der prinzipiellen Gestaltlosigkeit des Wassers. $\aleph^{26}$ Indem Dean auf künstliche Lichtquellen verzichtet, kann über die Dauer des Films

25 Peter Wollen, »Tacita Dean«, in: Afterall 1, 1999, S. 107-113, S. 109.

26 Ursula Frohne, »Motive der Zeit. Eine Anthologie von Zeitphänomenen in der Kunst der Gegenwart«, in: Heinrich Klotz (Hg.) Kunst der Gegenwart, Ausstellungskatalog Museum 
hinweg die Veränderung der Atmosphäre durch das Licht der untergehenden Sonne beobachtet werden. Das Licht steht in Deans Filmen symbolisch für die Tatsache, dass sie nur mit analogem, also lichtempfindlichem Film- und Fotomaterial arbeitet, was einen wesentlichen Aspekt ihrer Materialästhetik ausmacht - das Filmmaterial wird selbst zum schillernden Bild der Immaterialität des Lichts, als Dean es für Kodak (2006) während des Produktionsprozesses filmt. Der Leuchtturm in Disappearance at Sea (1996), auf Englisch lighthouse, fungiert als Allegorie der filmischen Lichtquelle, das Restaurant in Fernsehturm (2001) rotiert ebenso wie die Erde und erlebt den Wechsel von Tag zu Nacht, wodurch das goldene Licht der untergehenden Sonne zum Zwielicht und schließlich zur Dunkelheit wird und das optische Verhältnis von Innenund Außenraum sich langsam umkehrt. In The Green Ray (2001) wird die Suche nach dem >optisch Unbewussten $<$ das das Auge nicht sehen, der Film aber aufzeichnen und so sichtbar machen kann, motivisch umgesetzt: Der letzte Lichtstrahl der über dem Meer untergehenden Sonne soll aufgrund der atmosphärischen Beugung für einen kurzen Moment grün erscheinen; ob dies auf dem Film tatsächlich zu sehen ist, ist fraglich.

In der letzten Einstellung von Craneway Event scheint die Abendsonne durch die Pfeiler der Halle und schafft so ein räumlich anmutendes geometrisches Muster aus Licht, das die Tanzenden durchschreiten. Die natürliche Zeitlichkeit des Sonnenlichts, die den Rhythmus des Tages bestimmt, steht im Kontrast zu den jeweils individuellen Zeit-Räumen, welche die Tänzer/ innen im Training durch ihre Bewegungen definieren. Die für Cunningham charakteristischen »multiple[n] Zentren des Raumes, die sich ebenso durch die Position des Tänzers [...], in Bewegung oder im Stillstand, wie auch durch die Aussparung, die >leere< Raumzone herstellen $\ll, 27$ in Kombination mit der »tanzimmanenten Zeitgestalt $^{28}$ der trainierten Bewegungsabläufe zeigen sich in Deans Film als gleichwertig nebeneinanderstehend. So, wie der Film für Foucault selbst eine Heterotopie darstellt, da er über die zweidimensionale Projektion auf eine Leinwand einen dreidimensionalen Bildraum öffnet, so öffnet Dean mit Craneway Event einen Filmraum, der wiederum verschiedene Konzeptionen von Räumlichkeit und Zeitlichkeit zueinander in Beziehung setzt.

für Neue Kunst, ZKM Zentrum für Kunst und Medientechnologie Karlsruhe, Karlsruhe 1997, München / New York 1997, S. 38-5o, S. 41.

27 Gabriele Brandstetter, »Intervalle. Raum, Zeit und Körper im Tanz des 2o. Jahrhunderts«, in: Martin Bergelt, Hortensia Völckers (Hg.), Zeit-Räume. Zeiträume, Raumzeiten, Zeitträume, München/Wien 1991, S. 225-269, S. 25 o. 


\title{
Et in Utah Ego ... JG (2013)
}

\author{
»Everything about movies and moviemaking \\ is archaic and crude. One is transported by \\ this Archeozoic medium into the earliest \\ known geological eras.« \\ (Robert Smithson, The Spiral Jetty, 1972)
}

Das jüngste Filmprojekt Tacita Deans, das hier besprochen werden soll, beschäftigt sich mit einem Werk, das in der Geschichte der amerikanischen Kunst des 2o. Jahrhunderts eine herausragende Stellung einnimmt: Robert Smithsons Spiral Jetty (1970), die Ikone der Land Art und Bezugspunkt für zahlreiche zeitgenössische Künstlerinnen und Künstler. Spiral Jetty ist zum einen eine ortsbezogene Installation, die große spiralförmige, steinerne Mole im großen Salzsee in Utah, USA, wo Smithson sie 1970 mit schwerem Baugerät hat aufschütten lassen. Andererseits gehören zahlreiche Zeichnungen, Fotografien, ein Text (The Spiral Jetty, 1972) und der 32-minütige Film Spiral Jetty dazu, der die Entstehung der Mole dokumentiert und sie in einen historischen, geologischen und kosmologischen Kontext einbettet. Die Bedeutung des Kunstwerks im Rahmen des CEuvres von Smithson und darüber hinaus für die Land Art kann nicht überschätzt werden. Durch ihre jahrelange Nicht-Sichtbarkeit Spiral Jetty war, bedingt durch den Wasserstand des Salzsees, von 1972 bis 2002 nicht zu sehen - erlangte die Arbeit geradezu mythische Bedeutung als Fixpunkt einer Bewegung innerhalb der modernen Kunst, die konzeptuelles Denken mit Ortsspezifik und der Sensibilität für das Verhältnis von Mensch, Natur, Technik und Wissenschaft verknüpft. Tacita Dean hat bereits 1997 den Versuch unternommen, anhand von Smithsons Wegbeschreibungen Spiral Jetty zu finden, scheiterte jedoch und verarbeitete die Reise in einer Klangarbeit. Von dieser Arbeit Trying to Find Spiral Jetty (1997) berichtete der Kurator Jeremy Millar dem Science-Fiction-Autor J. G. Ballard, wodurch eine Korrespondenz zwischen Tacita Dean und Ballard über Smithsons Arbeit in Gang gesetzt wurde. Millar erkannte die Parallelen zwischen Ballards Kurzgeschichte The Voices of Time (1960), die Smithson in seinem Besitz hatte, und Spiral Jetty. »Firstly, why a spiral jetty? What conceivable cargo would require a spiral jetty for its loading/unloading? «, ${ }^{29}$ fragt Ballard Dean in einem Brief vom 22. Oktober 2007, und fordert sie später auf: »Treat it as a mystery that your

29 James Graham Ballard, Brief an Tacita Dean vom 22.10.2007, in: Richard Torchia (Hg.), JG. a film project by Tacita Dean, Ausstellungskatalog Arcadia University Art Gallery, Spruance Arts Center, Glenside 2013, S. 12. 
film will solve. ${ }^{30}$ Ballards und Deans gemeinsames Interesse für Smithson gab somit den Anstoß für das Filmprojekt $J G$, welches einen Dreiklang zwischen der Arbeit von Smithson, der Kurzgeschichte The Voices of Time von Ballard und ihrer eigenen filmischen Tätigkeit zu visualisieren sucht. Deans spezifische Bildsprache und die Technik der Collage und des Maskierens, welche sie an FILM entwickelt hat, werden eingesetzt, um Smithsons Werk neu zu >rahmen< und seine historische Bedeutung für die zeitgenössische Filmkunst herauszuarbeiten. Wie gelingt ihr dies, und welches Geschichtsbild zeigt sich aus der Konfrontation von Spiral Jetty und der Literatur von Ballard? Welche narrativen Mittel setzt sie ein, und wie werden dadurch verschiedene Zeitkonzepte mit der Geschichtlichkeit der Werke, aber auch der Landschaft, verwoben? Welche Rolle schließlich spielt die Zeit für alle drei Elemente der Arbeit?

Tacita Deans Film $J G$ ist 26 1/2 Minuten lang, er wurde auf $35 \mathrm{~mm}$ in Farbe und in Schwarz-Weiß mit einer anamorphotischen Linse und optischer Soundaufnahme an verschiedenen Orten in Utah und Kalifornien gedreht und wird im Loop in einem abgedunkelten Raum mit Stuhlreihen für die Zuschauer gezeigt. Das Voiceover mit Auszügen aus Ballards The Voices of Time (1960), Prisoner of the Coral Deep (1964), Robert Smithson as Cargo Cultist (1997) und der Korrespondenz zwischen Ballard und Dean spricht der Schauspieler Jim Broadbent. In Deans Film sind zunächst zwei formale Besonderheiten auffällig: Zum einen benutzt sie die bereits in FILM (2011) erprobte und patentierte Technik des Maskierens, um mittels eigens erstellter Blendmasken, welche die anamorphotische Verzerrung des Breitbildformats ausgleichen können, durch aufwändige Mehrfachbelichtung verschiedene Aufnahmen und Formen bereits in der Kamera nach Art einer Collage ineinander- oder übereinanderzumontieren. ${ }^{31}$ Zum anderen rahmt sie einige Aufnahmen, ebenfalls wie in FILM, mit einer Art schwarzem Lochfilmstreifen, der in drei Teile unterteilt ist, so dass der Eindruck entsteht, man sähe drei in sich bewegte Einzelbilder eines Films. Dies erlaubt die Kombination von drei Aufnahmen in einem projizierten Bild, die wie in einer Mehrfachprojektion nebeneinanderstehen und von den Betrachtenden zueinander in Beziehung gesetzt werden. In

$30 \quad$ Tacita Dean, $» \mathrm{~J}$ «, in: Torchia, JG. a film project by Tacita Dean, S. 15 .

$31 \mathrm{Zu}$ FILM und die darin erstmals angewandte Technik siehe den Katalog zur Ausstellung in der Tate Modern, London: Cullinan (Hg.), Tacita Dean. Film, sowie u. a. meine Ausführungen dazu (Nina Steinmüller, »FILM als Film. Bildbewegungen bei Tacita Dean«, in: Rathgeber, Steinmüller, BildBewegungen/ImageMovements, S. 179-200). 
diesen Einstellungen wechseln sich Farb- und Schwarz-Weiß-Aufnahmen ab, die jedoch aufgrund des für Dean typischen breiten Formats besonders mit der Horizontalität der gefilmten Landschaften korrespondieren.

Die Struktur des Films ähnelt, wie Dean in einem kurzen Interview erläutert, der von The Voices of Time, denn er besteht aus fünf kürzer werdenden Abschnitten, die jeweils mit Aufnahmen einer Uhr unterteilt sind - wie beim Protagonisten der Kurzgeschichte, dessen Wachphasen sich Schritt für Schritt verkürzen. Diese Analogie, betont Dean, war nicht geplant und ist für ihre Arbeitsweise auch unüblich, ${ }^{32}$ zeigt aber dennoch, wie eng ihre filmische Arbeitsweise mit der Zeitstruktur der Erzählung und auch der Skulptur beziehungsweise Smithsons Film verwoben ist, wie wir weiter unten sehen werden. Bevor auf $J G$ näher eingegangen wird, sollen daher die Werke von Smithson und Ballard kurz erläutert werden.

Robert Smithsons umfangreiches CEuvre, das durch seinen plötzlichen Unfalltod im Alter von 35 Jahren 1973 ein jähes Ende findet, entwickelt sich aus der Minimal Art heraus. Stärker als andere Land-Art-Künstler fundiert und reflektiert er seine Erdarbeiten, die seit den 196oer Jahren entstehen, in seinen Texten, die sich zwischen literarischen Essays und theoretischen Reflexionen über Geschichte, Kunstgeschichte, Physik, Geologie, Biologie und Astronomie bewegen. ${ }^{33}$ Die Zeit - als Zeit der künstlerischen Erfahrung, als Geschichtlichkeit von Dingen, Materialien und Stoffen - steht dabei im Mittelpunkt seines Interesses und wird an Begriffe und Konzepte wie Entropie, Monument oder Museum geknüpft. In »Entropie und neue Monumente« (Artforum, Juni 1966) diskutiert Smithson die Werke einiger zeitgenössischer MinimalArt-Künstler wie Donald Judd, Robert Morris, Sol LeWitt und Dan Flavin als neue Monumente, die nicht der Erinnerung an die Vergangenheit, sondern dem Vergessen der Zukunft dienten. ${ }^{34}$ Nicht die Zeit in ihrem herkömmlichen Sinn als historische Entwicklung, biologische Evolution oder die Zeitalter überdauernde Einheit, in natürlichen Materialien repräsentiert, beschäftige diese Künstler, sondern ihre Werke reduzierten die Zeit auf den Moment der Erfahrung des Ortes und der neuen, künstlichen Materialien:

32 »Tacita Dean: On JG and the Medium of Time«, 28.2.2013, filmed at the Pew Center for Arts and Heritage, https://www.pewcenterarts.org/post/tacita-dean-jg-and-mediumtime [26.4.2020].

33 Robert Smithson, The Collected Writings, Berkeley 1996, auf Deutsch erschienen als: Robert Smithson, Gesammelte Schriften, hg. v. Eva Schmidt, Kai Vöckler, Köln 2000.

34 Robert Smithson, »Entropie und Neue Monumente« (1966), in: ders., Gesammelte Schriften, S. 27-37, S. 28. 
Vergangenheit und Zukunft werden beide in einer objektiven Gegenwart plaziert. Diese Art von Zeit hat wenigen oder gar keinen Raum, sie ist stationär und bewegungslos, sie geht nirgendwohin, sie ist eine un-newtonsche Zeit und sie besteht im Augenblick, und sie ist gegen die Räderwerke der Zeit-Uhr. [...] Flavins Dekonstruktion der klassischen Zeit und des klassischen Raums beruht auf einer völlig neuen Auffassung von der Struktur der Materie. ${ }^{35}$

Schlüsseltopos für diese Zeitlosigkeit ist die Entropie, der zweite Hauptsatz der Thermodynamik, der besagt, dass in einem geschlossenen System aufgrund der Irreversibilität der meisten Prozesse die Menge der Unordnung, das heißt der während eines Prozesses abgegebenen Energie, stetig zunimmt und so theoretisch das Universum eines Tages zum Stillstand durch Wärmetod kommen wird. Dieses die gesamte Physik überspannende Prinzip sieht Smithson in der Kunst der Minimal Art »»zelebriert« und er charakterisiert diese Werke somit als abgeschlossene, zeitlose Systeme. ${ }^{36}$ Smithson versucht in seinen eigenen ortsbezogenen Arbeiten der Entropie in Form der Umweltzerstörung und Energievernichtung symbolisch entgegenzuwirken. Seine Werke weisen auf die Unumkehrbarkeit der menschlichen Eingriffe in die Natur hin und sollen so die begrenzten Zeithorizonte menschlichen Handelns relativieren. Seine architektonischen Projekte dieser Zeit stehen unter dem Konzept der »De-Architekturierung «, dem »Bauen unter dem Aspekt einer unausweichlichen entropischen Entwicklung «.37 Dies meint eine »Architektur der Vorläufigkeit, die ihr eigenes Verschwinden thematisiert« angesichts der Tatsache, »dass der entropische Prozess unweigerlich in eine bildlose Ewigkeit führt, hin zu einem Endzustand ohne Veränderung und Entwicklung «. ${ }^{38}$ Smithsons 1967 unternommener Ausflug zu seinem Heimatort Passaic in New Jersey, dessen Industriebrachen er fotografisch festgehalten hat, hat seinen Begriff von Geschichte nachhaltig verändert. Hier findet er die postindustriellen Monumente des zeitgenössischen Amerika, das nicht wie Rom auf eine antike Geschichte zurückgreifen kann, sondern die Prozesse des Erinnerns an den Bruchkanten einer Naturgeschichte und den Ruinen der modernen Zivilisation verorten muss. Verlassene Maschinen scheinen im Matsch gefangen zu sein wie mechanische Dinosaurier, ${ }^{39}$ die Stadt beschreibt

\section{Ebd.}

36 Ebd., S. 27.

37 Kai Vöckler, »Das Verschwinden der Architektur als Thema der Kunst«, in: Sabine Folie (Hg.), Die Moderne als Ruine. Eine Archäologie der Gegenwart, Ausstellungskatalog Generali Foundation, Wien 2009, Nürnberg 2009, S. 13-17, S. 15.

38 Ebd.

39 »Weil es Samstag war, waren viele Maschinen nicht in Betrieb, und das ließ sie wie im Schlamm steckengebliebene prähistorische Kreaturen aussehen oder mehr noch wie 
er als ruinös, aber nicht im romantischen, erhabenen Sinn des Verfalls, sondern zeitlich verkehrt:

Dieses Null-Panorama war offenbar voller umgekehrter Ruinen - voller neuer Bauten, die hier einmal hingesetzt werden würden. Umgekehrte Ruinen sind das Gegenteil der >romantischen Ruinen`, denn diese Bauten zerfallen nicht in Trümmer, nachdem sie gebaut wurden, sondern erheben sich zu Trümmern, bevor sie gebaut wurden. Diese anti-romantische Inszenierung verweist auf die diskreditierte Idee der Zeit und viele andere sveraltete Dinge. ${ }^{40}$

Smithson erweitert in seinen Werken und theoretischen Reflexionen Begriffe wie die Ruine oder das Monument und löst sie von ihrer ursprünglichen kunsthistorischen Bedeutung, indem er sie auf die postindustrielle Gegenwart der 1960er Jahre bezieht; er zieht Verbindungslinien von prähistorischen Symbolen und Bauwerken bis in die Zukunft und offenbart dabei ein abstraktes Zeitverständnis, das nicht an lineare Zeitvorstellungen gebunden ist.

Die Land-Art-Skulptur Spiral Jetty (Abb. 27, 28) entstand 1970, nachdem Smithsons Interesse für die geologischen Besonderheiten von Salzseen ihn zunächst an den Mono Lake in Kalifornien geführt und zu Recherchen über die Salzseen in Bolivien angeregt hatte, wie er in dem Text »Spiral Jetty« beschreibt. Der Große Salzsee in Utah wurde aufgrund seiner zum Teil roten oder rosa Färbung für eine Erdarbeit ausgewählt. Die Skulptur, welche Smithson aus Basaltgestein und Erde als gegen den Uhrzeigersinn verlaufende Spirale auftürmen ließ, ragt ca. 46o Meter in den See hinein und ist ca. 4,5 Meter breit. Sie ist je nach Wasserstand für Besucher/innen sichtbar und begehbar oder unter der Wasseroberfläche verborgen. Die in Film und Essay so gradlinig beschriebene und dokumentierte Errichtung hat, wie Michael Lüthy erläuterte, auch fiktionale Anteile, da der Entwurf ursprünglich eine zum Ufer zurückgebogene Mole mit einer Plattform am Ende vorsah. ${ }^{41}$ Erst nach Beendigung der Arbeiten entschied sich Smithson, die Form in die einer Spirale zu ändern und wählte so ein einprägsames Bild für die im Film visualisierten vielfältigen makro- und mikrokosmologischen Zusammenhänge.

ausgestorbene Maschinen - abgehäutete mechanische Dinosaurier.« (Robert Smithson, »Fahrt zu den Monumenten von Passaic, New Jersey« [1967], in: ders., Gesammelte Schriften, S. 97-102, S. 99.)

40 Ebd., S. 100.

41 Vgl. Michael Lüthy, »Das falsche Bild. Robert Smithsons verworfene Erstversion der Spiral Jetty«, in: Sebastian Egenhofer, Inge Hinterwaldner, Christian Spies (Hg.), Was ist ein Bild? Antworten in Bildern. Gottfried Boehm zum 70. Geburtstag, München 2012, S. 279-281, S. 279. 


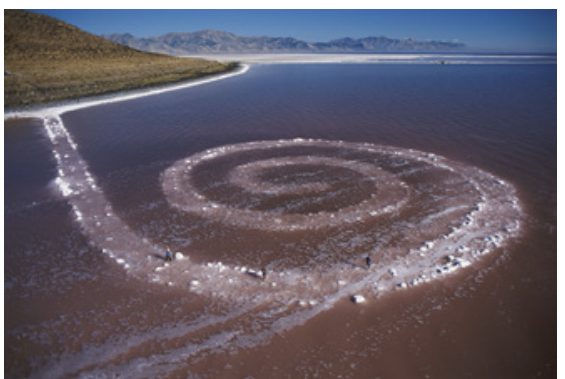

Abb. 27
Robert Smithson, Spiral Jetty, 1970, Foto: George Steinmetz

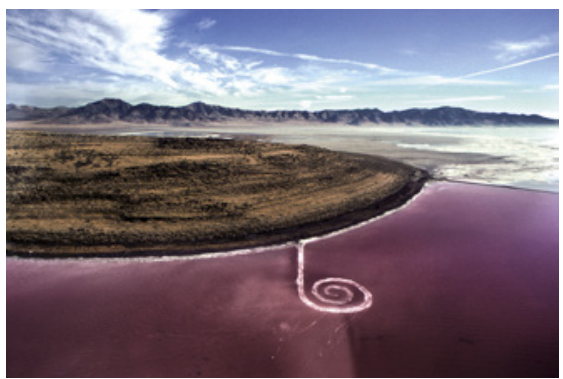

Abb. 28
Robert Smithson, Spiral Jetty, 1970, Foto: Nancy Holt

Der Film The Spiral Jetty, welcher parallel zur Erstellung der Skulptur gedreht wurde, ist keine Dokumentation des Projekts, sondern stellt eine eigene visuelle Reflexion der Erdarbeit, ihrer spiralförmigen Form und der geologischen und historischen Einbettung in die Landschaft dar. Er beginnt mit Bildern von Gasexplosionen auf der Sonne, gefolgt von der Suche nach dem geeigneten Ort für die Mole am Ufer des Großen Salzsees, Ausschnitten von Karten und rot eingefärbten Aufnahmen von Dinosaurierskeletten aus einem naturhistorischen Museum - ein Panorama aus Bildsequenzen, die den Hintergrund für die Errichtung der Mole darstellen:

Der Film rekapituliert die Skala der Spiral Jetty. Disparate Elemente geraten in Zusammenhang. Unwahrscheinliche Orte und Dinge sind zwischen Szenen montiert, die ein Stück einer unbefestigten Straße zu dem realen Ort in Utah und zurück zeigen. [...] Der trennende Unterschied zwischen Realität und Film erzeugt das Gefühl eines kosmischen Bruchs. ${ }^{42}$

Im weiteren Verlauf kontrastiert Smithson Aufnahmen der Erstellung der Mole (Smithson, wie er im Wasser watend Entfernungen abmisst, Bagger und Lastwagen, wie sie Erde, Basalt und Steinmassen an Land aufnehmen, ins Wasser schütten und so zur Spirale türmen) mit Aufnahmen der rosafarbenen Wasseroberfläche und zwei Bildern von Dinosauriern beziehungsweise einer Echse. Schließlich folgen als dritter Teil die aus einem Hubschrauber gefilmten Aufnahmen der Mole, auf der Smithson nach innen läuft. Der Hubschrauber umkreist das Bauwerk und nähert sich ihm immer wieder aus verschiedenen Richtungen. Auf der Tonebene wechseln sich sehr laute Sequenzen mit

42 Robert Smithson, »Spiral Jetty« (1972), in: ders., Gesammelte Schriften, S. 176-184, S. 183. 
Bau- beziehungsweise Helikopterlärm mit sehr stillen Sequenzen ab, darüber liegt das von Smithson gesprochene Voiceover. ${ }^{43}$

The Voices of Time ist eine dystopische Kurzgeschichte von James Graham Ballard, die 196o entstand und, wie auch andere Bücher Ballards dieser Periode, von der Zeit handelt. Hauptperson ist Robert Powers, ein Neurochirurg, der an einem heißen, nicht näher benannten Ort an einer Klinik für sogenannte sleepers arbeitet und gleichzeitig das Labor seines durch Selbstmord verstorbenen Kollegen Whitby betreut. Er stellt an sich die Anzeichen einer fortschreitenden Degeneration fest, wie sie auch seine Patienten aufweisen, die sich aufgrund eines zusätzlichen, inaktiven Chromosomenpaars, die silent pairs, nach und nach körperlich und mental zurückentwickeln und vermehrt schlafen, bis sie schließlich nicht mehr aufwachen und in einen komatösen Zustand verfallen. Powers möchte die ihm verbliebenen wachen Stunden noch sinnvoll nutzen und führt Tagebuch. In Whitbys Labor hatten sie versucht, durch Aktivierung der silent pairs bei bestimmten Tieren und Pflanzen mittels Röntgenbestrahlung deren verborgene Entwicklungsmöglichkeiten freizusetzen, und tatsächlich weisen einige so Behandelte anschließend erstaunliche Fähigkeiten auf, die jedoch am Ende auch zur Selbstzerstörung führen. Powers wird von einem ehemaligen Patienten verfolgt, Kaldren, der aufgrund eines missglückten Experiments überhaupt nicht mehr schläft und in einem spiralartig-verdrehten Betonhaus am Ufer eines Salzsees wohnt. ${ }^{44}$ Kaldren ist besessen von Zahlencodes, die er angeblich aus dem Weltraum empfängt und die das bevorstehende Ende der Welt zeitlich ankündigen, wie er Powers anhand von mehreren Funkempfängern vorführt, die einen numerischen Countdown herunterzählen. Powers versucht, ein Mandala, das Whitby in einen ausgetrockneten Swimmingpool gezeichnet hat, in Beton nachzubauen. Schließlich begibt er sich selbst zur Röntgenbestrahlung ins Labor und kehrt anschließend zu dem Mandala zurück, wo er auf der mittigen Plattform in einer Art Verschmelzung mit der kosmischen Zeit der Sterne stirbt. Er wird von Kaldren und dessen Freundin Coma gefunden. Kaldren fährt noch ein letztes Mal zum Labor, in dem alle Versuchstiere und -pflanzen gestorben sind. Er informiert die Polizei über Powers Tod, nimmt eine Filmrolle, die er dort findet, mit, und zieht sich in sein Haus zurück.

43 Vgl. zum Ablauf des Films auch Diane Thater, »A Man Becomes Unstuck in Time in the Film that Became a Classic!«, in: Lynne Cooke, Karen Kelly (Hg.), Robert Smithson Spiral Jetty. True Fictions, False Realities, Berkeley 2005, S. 165-183.

44 Die Beseitigung des Schlafbedürfnisses durch einen chirurgischen Eingriff mit jedoch negativen Folgen für den Patienten geht auf eine andere Kurzgeschichte Ballards zurück, Manhole 69 von 1957 . 


\section{Besonderheiten JG}

Tacita Deans Werk orientiert sich in mancherlei Hinsicht an Smithsons Film Spiral Jetty, es fallen jedoch zunächst die Unterschiede ins Auge. Wo Spiral Jetty einer erzählenden Dramaturgie folgt - der Suche und Erschließung des geeigneten Standorts, dem Bau der Spirale und der Begehung durch Smithson, was vom Helikopter aus gefilmt wird, schließlich der Entstehung des Films im Studio -, erscheint $J G$ stärker als eine nichtnarrative Kontemplation über die Besonderheiten der Landschaft, in welcher Spiral Jetty und The Voices of Time angesiedelt sind. $J G$ hat kein erzählendes Voiceover, sondern nur wenige gesprochene Sätze begleiten die meist langen, statischen Einstellungen. Es wechseln sich in loser Folge Aufnahmen aus der Umgebung des Großen Salzsees, von den Uferbereichen, von dort arbeitenden Maschinen und Turbinen, von den Bergen und wüstenähnlichen Landschaften ab, die Dean unter anderem im Death Valley aufgenommen hat. Licht und Wasser, die gekräuselte Wasseroberfläche, Spiegelungen und Brechungen sind wie schon in früheren Filmen Deans und auch bei Spiral Jetty die dominierenden Bildmotive. In den Sequenzen, die das Bild mittels maskierter Lochstreifen in drei nebeneinanderstehende Einstellungen unterteilen, kombiniert Dean Aufnahmen von arbeitenden Baggern oder Turbinen, von Salzkristallen und immer wieder von Details zweier kleiner Gürteltiere, die sich einrollen, sowie von einer Echse (Abb. 29). ${ }^{45}$ Einzelne Einstellungen mit kristallinen Strukturen oder aufgetürmten, weißen Felsen lassen den Betrachter / die Betrachterin bezüglich ihrer Maßstäblichkeit im Unklaren und öffnen das Bild so für die Wahrnehmung von Oberflächen, Strukturen und Kontrasten zwischen den salinen und steinernen Objekten und den wechselnden Schattierungen des Himmels. ${ }^{46}$ Wie in dem Film Spiral Jetty entsteht so eine Zusammenschau von Makro- und Mikrokosmos der besonderen Landschaft um den Großen Salzsee und die Erdskulptur; eine Beschreibung Mark Glödes von Smithsons Film könnte ohne Weiteres auch auf $J G$ bezogen werden:

45 In Smithsons Film taucht als Standbild die Fotografie einer Echse auf; das Gürteltier wird in The Voices of Time erwähnt, als Powers ein kleines gepanzertes, unbestimmbares Tier findet, das ihn an ein Gürteltier erinnert.

46 Auch Smithson verweist in seinem Text zu Spiral Jetty auf die Relativität der Maßstäblichkeit, die bereits die Skulptur selbst und ihre Wahrnehmung betrifft und im Film aufgegriffen wird: »Solange man sich nicht darauf einläßt, die Maßstäblichkeit unabhängig von den Ausmaßen zu sehen, hat man nur ein Objekt oder eine Formulierung, die eindeutig zu sein scheinen. [...] Jeder kubische Salzkristall bildet in seinem Molekulargitter ein Echo der Spiral Jetty.« (Smithson, »Spiral Jetty«, S. 179.) 


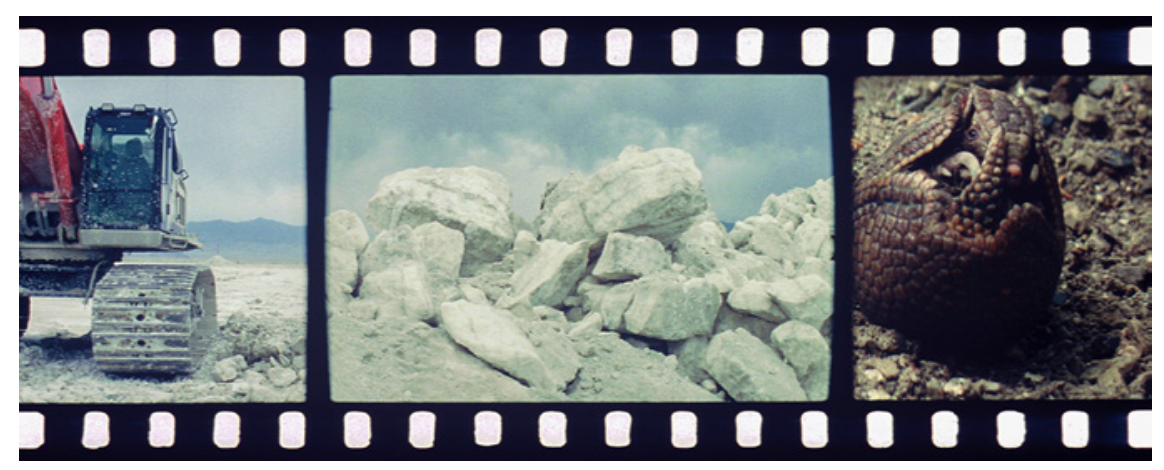

Abb. 29 Tacita Dean, $J G, 2013$, Filmstill

Mit seinem Film dringt Smithson somit anders als bisher gekannt in die amerikanische Wüste ein. Indem er kartographische Blicke mit mikroskopischen Eindrücken verbindet, führt er im Film unmittelbar das Nahe mit dem Entfernten zusammen. Mikroskopische Nahaufnahmen von Mineralien, Salzkristallen oder Großaufnahmen von ins Wasser fallenden Gesteinsbrocken werden kombiniert mit panoramatischen Ansichten einer Landschaft oder Aufsichten auf Teile der aufgeschütteten Spirale. ${ }^{47}$

Ein entscheidender Unterschied ist, dass Dean die Geschichte von Spiral Jetty nicht auf deren Entstehung bezieht, sondern die Erdarbeit als bereits historisches Monument der Land Art in Form eines Erinnerungsbildes in der Collage zitiert. Die tatsächliche Skulptur wird im Film JG nicht gezeigt, jedoch als Maske verwendet (Abb. 3o) und findet sich als universale Form der Spirale in Wasserwirbeln oder den rollenden Bewegungen des Gürteltiers wieder. Das Wasser als Fluss der Zeit treibt die sich konstant drehenden Turbinen an, die wie archaische Mühlräder das Wasser von einem Becken ins andere schaufeln. Die Spiralform, als Symbol eine der ältesten Formen, die sich weit verbreitet in der Kunst zeigt, manifestiert sich makrokosmisch in den Spiralnebeln unseres Sonnensystems, mikrokosmisch in wirbelndem Wasser ebenso wie in natürlichen Formen wie Farnen oder Schneckenhäusern. ${ }^{48}$ Mit der Spirale verbindet Smithson, wie Anne Hoormann ausführt, ein »schematisches Bild

47 Marc Glöde, »Filmische Topographien der USA. Zur Konstruktion von Landschaften in der experimentellen Filmgestaltung «, in: Augen-Blick 37: Blicke auf Landschaften, Marburg 2005, S. 25-45, S. 36.

48 Vgl. »Spirale«, in: Hans Biedermann, Knaurs Lexikon der Symbole, München 1998, S. $416-418$. 


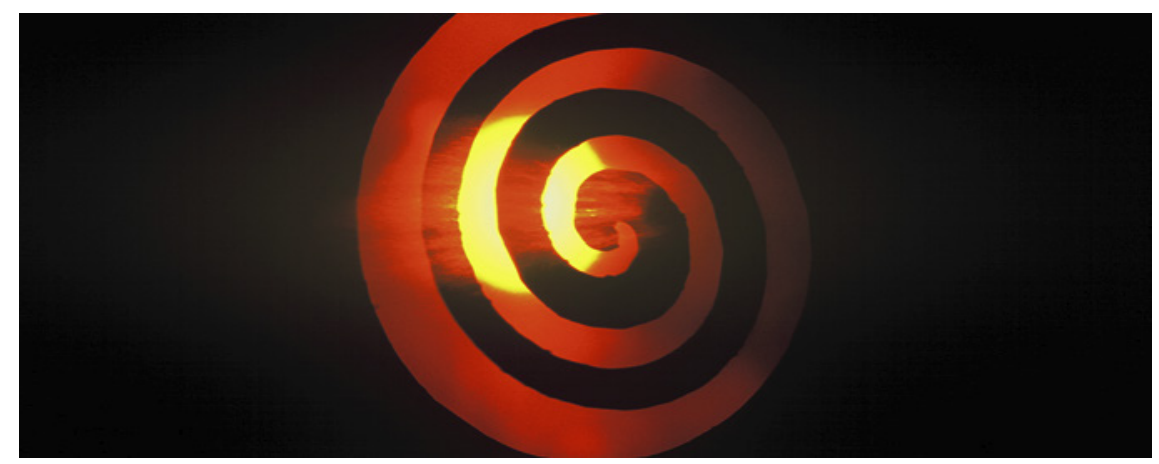

Abb. $30 \quad$ Tacita Dean, $J G, 2013$, Filmstill

für die Entwicklung des Universums«.49 Er verknüpft den mathematischen Ursprung der Spirale, ihre Berechenbarkeit, mit der Irrationalität ihrer Erfahrung. Smithsons Fortschrittskritik, die entropische Logik eines zwangsläufigen Wärmetods, zeigt sich in dem Symbol, das für die Rückkehr zum Ursprung steht: »[M]it jedem Schritt auf der Spirale kehren wir zu unserem Ursprung zurück. «50 Die Arbeit in der Wüste ist so auch eine geschichtliche, archäologische Arbeit, die historisches, kosmologisches und naturwissenschaftliches Wissen miteinander verknüpft.

\section{Landschaften}

Betrachtet man die Landschaft vom Blickpunkt der Ästhetik aus, ist hier die Natur »im Anblick für einen fühlenden und empfindenden Betrachter ästhetisch gegenwärtig «. ${ }^{51}$ Landschaft hebt sich als ein gewählter Ausschnitt aus der in ihrer Totalität nicht greifbaren Natur als wiederum Ganzes hervor. ${ }^{52}$ Im narrativen Film hat die Landschaft häufig eine konkrete Zuschreibung beziehungsweise Aufgabe, sie ist Projektionsfläche für die Gefühlswelt der filmischen Figuren, Synonym für Heimat oder Fremde, für Identität oder Verlust derselben, sie macht durch Wetterphänomene Stimmungen und Zeitverläufe deutlich oder hat als konkreter Schauplatz eine historische Bedeutung,

49 Anne Hoormann, Land Art. Kunstprojekte zwischen Landschaft und öffentlichen Raum, Berlin 1996, S. 84.

50 Smithson, »Spiral Jetty«, S. 180.

51 Joachim Ritter, Landschaft. Zur Funktion des Ästhetischen in der modernen Gesellschaft, Münster 1963, S. 18.

52 Vgl. Georg Simmel, »Philosophie der Landschaft« (1913), in: ders., Aufsätze und Abhandlungen 1909-1918, Band 1, hg. v. Rüdiger Kramme, Angela Rammstedt, Frankfurt am Main 2001, S. 471-482. 
auf die im Film angespielt wird. Sergej Eisenstein hat beschrieben, dass mit Blick auf ihre Ausdrucksmöglichkeiten die Landschaft der Musik am nächsten komme, dass die Landschaft das »unabhängigste Element des Films «53 sei: »[S]ie ist am wenigsten belastet von bedienenden-erzählerischen Aufgaben und am anpassungsfähigsten bei der Wiedergabe von Stimmungen, emotionalen Zuständen und seelischen Vorgängen, mit einem Wort, all dessen, was in seiner vage erfaßbaren, fließenden Bildhaftigkeit eigentlich nur durch Musik ausdrückbar ist. «54 In jedem Fall aber ist Landschaft eine »kulturelle Konstruktion $\ll, 55$ die immer auch den Blickwinkel des/der Betrachtenden ausdrückt, sie »fixiert Vorstellungen unserer Umwelt und sozialen Umgebung «. ${ }^{6}$ Insofern ist sie auch immer ein Ausweis der Historizität des filmisch eine Landschaft darstellenden, rahmenden Subjekts.

Tacita Dean hat schon in früheren Arbeiten Landschaften dargestellt, insbesondere die englischen Küsten in ihren Leuchtturmfilmen oder in Banewl. In letzterem Film dokumentierte sie auf einer Milchfarm in Cornwall die Sonnenfinsternis von 1999, welche aufgrund des bedeckten Himmels jedoch nur als sehr allmähliche Verdunkelung und Wieder-Aufhellung der ländlichen Szenerie wahrgenommen werden kann. Deans Verhältnis zur Natur ist in ihren Filmen von einem starken Interesse an natürlichen Zeitabläufen, Licht- und Wetterphänomenen gekennzeichnet. Sie verwendet selten Schnitte, um das Gefühl für das Verstreichen der Zeit und ihre Bannung mittels des Lichts auf den Film zu erhalten. Indem kosmische Abläufe wie die Sonnenfinsternis oder auch die untergehende Sonne in The Green Ray als Kräfte dargestellt werden, die unsere natürlichen Zeitverläufe auf der Erde seit jeher beeinflussen und unsere Zeitwahrnehmung strukturieren, verzahnen sich verschiedene Zeitrechnungen miteinander und wird die Zeit als das eigentliche Thema ihrer filmischen Arbeit deutlich. So auch in JG, wo Dean sich wie Smithson der Wüstenlandschaft um den Großen Salzsee in Utah widmet, dies aber unter völlig veränderten historischen und ästhetischen Vorzeichen. Smithsons Essay und Film dokumentieren, wie der Standort der Mole aufgrund landschaftlicher Kriterien ausgewählt wurde: Die Rotfärbung des Wassers im nördlichen Teil des Sees als Folge bakterieller Zersetzungsprozesse und Algenbildung interessierte ihn, und Smithson berichtet von einem nahezu erhabenen Landschaftseindruck beim ehemaligen Ölfeld Rozel Point, wo die Wasseroberfläche

53 Sergej M. Eisenstein, Eine nicht gleichmütige Natur, hg. v. Rosemarie Heise, Berlin 1980, S. 16.

54 Ebd.

55 Barbara Pichler, Andrea Pollach, »Vorwort«, in: dies. (Hg.), Moving landscapes. Landschaft und Film, Wien 2006, S. 9-14, S. 11.

56 Ebd. 
und die untergehende Sonne miteinander verschmelzen und den Eindruck einer unbewegten Bewegtheit heraufbeschwören, die in der Form einer Spirale kulminiert:

\begin{abstract}
Als ich diesen Ort sah, vibrierte er bis an den Horizont; er erschien wie ein immobiler Zyklon, und das flimmernde Licht erweckte den Eindruck, als würde die ganze Landschaft beben. Ein latentes Erdbeben verbreitete sich in der zitternden Stille, wurde zu einer kreisenden Empfindung ohne Bewegung. Der Ort begann zu rotieren, umschloß sich selbst in einer immensen Rundung. Aus diesem kreisenden Raum tauchte die Möglichkeit der Spiral Jetty auf. ${ }^{57}$
\end{abstract}

Bei Dean stehen nicht das Bauwerk und seine Errichtung, sondern seine Einbettung in die es umgebende Landschaft im Mittelpunkt. So widmen sich viele Einstellung in der Totalen der Berglandschaft und den Wolkenformationen, die im Licht der untergehenden Sonne erst in Rot- und Orangetönen leuchten, dann im blauen Licht der Dämmerung und schließlich in der Nacht versinken. Die Sonne, die als erstes Bild des Films über dem an einen Swimmingpool erinnernden Parallelogramm erscheint - man denkt an den Pool in The Voices of Time - taucht auch in JG immer wieder auf, stets untergehend, so dass sie als Anspielung auf die untergehende Welt der Erzählung gesehen werden kann $\left(\right.$ Abb. 31). ${ }^{58}$

Zur Landschaft bei Smithson stellt Mark Glöde fest, dass sich hier andere Diskursfelder angelagert hätten und diese Ebenen sich nicht homogen zueinander verhielten: »Die einzelnen Splitter ergeben keine geordnete Landschaft

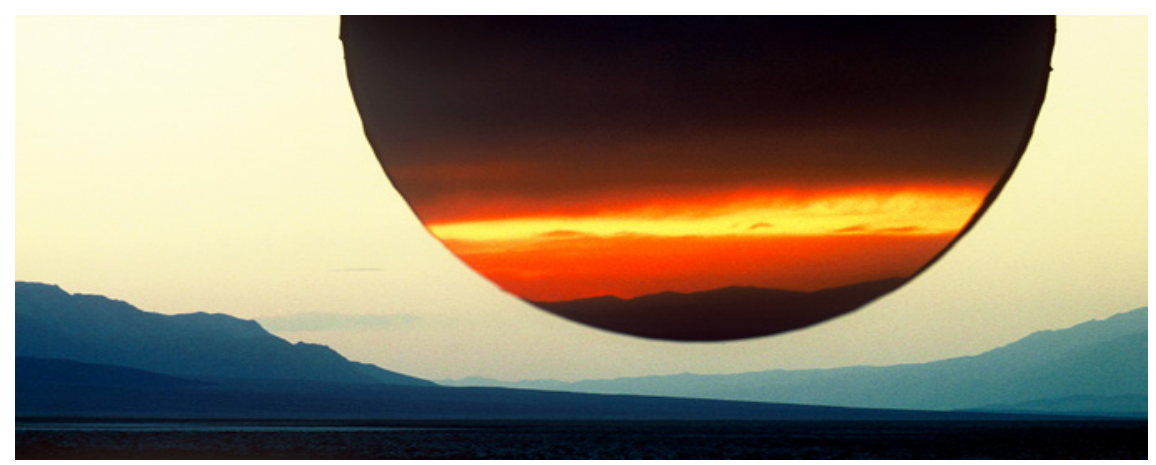

Abb. 31 Tacita Dean, $J G, 2013$, Filmstill

57 Smithson, »Spiral Jetty«, S. 179.

$5^{8}$ Die Form der Maske findet sich auch in Smithsons Fotoserie zu Monuments of the Passaic, wo ein von der Seite fotografierter Sandkasten die Form eines Parallelogramms annimmt. 
mehr und auch kein größeres Raumsystem, in das sich diese Landschaft mathematisch wie ein Puzzle zusammenfügen könnte, sondern sie verweisen auf einen dynamischen und entropischen Prozeß.«59 Ebendies kann auch bei $J G$ beobachtet werden. Dean kombiniert Anspielungen auf Science-FictionThemen mit Aufnahmen der industriellen Salzverarbeitung, der großen Baumaschinen, die den Raubbau an der Natur symbolisieren. Bagger, Walzen, Züge - alle Geräte scheinen wie von selbst zu funktionieren, die Szenen sind menschenleer. Dean kreiert in JG Bilder des leeren Raums und evoziert durch die eingefügten Formen, die an Planeten, Monde und die Sonne erinnern, sowie die schematisierte Spirale Assoziationen an Topoi der Science-FictionLiteratur. Die Natur, die hier gezeigt wird, ist in keiner Weise fruchtbar: Die Salzwüste Utahs ist eine der unwirtlichsten Gegenden der USA, nur wenige Tier- und Pflanzenarten sind an die kargen Böden und das extreme Klima angepasst. Ballards anschauliche Beschreibungen der kargen Landschaft hat Dean in ihrem Film in Bilder übersetzt: den Kontrast des weißen, flachen Sees und der hoch aufragenden Hügel im Hintergrund, die spärliche Vegetation und die lebensspendende, aber auch potenziell tödliche Strahlung der Sonne und der Sterne. Ballard greift das Prinzip der Anpassung an veränderte Lebensumstände in seiner Erzählung mit dem Bild des gürteltierähnlichen kleinen Tieres auf, dem ein bleierner Panzer gewachsen ist, der es vor kosmischer Strahlung schützen könnte. Powers findet dieses Tier und bringt es zu Untersuchungszwecken in das Labor. Das bevorstehende Ende der Erde beziehungsweise ihrer Bewohner ist bei ihm schon angezählt. Dean erschafft ebenso wie Ballard in seiner Geschichte Bilder der landschaftlichen Leere als eine Vision der Erde, die am Ende des entropischen Prozesses ohne Menschen weiterexistieren wird.

\section{Zeitstrukturen}

Es sind zwei Erscheinungsformen der Zeit, die in den Werken der drei Künstler im Vordergrund stehen: die Entropie und die Parallelität verschiedener Zeiten im Bild. Die Entropie als zentrale Kategorie des Schaffens von Smithson spielt bei Ballard, der diese als Erster in die Science-Fiction-Literatur eingebracht hat, eine ebenso große Rolle, wie Michael K. Iwoleit beschreibt:

In >The Voices of Time $<$ (196o, dt. >Die Stimmen der Zeit $<$ ), neben >The Terminal Beach möglicherweise Ballards bedeutendster Beitrag zur Science Fiction, taucht Zeit schließlich in Gestalt der Entropie, des Verfalls, der Degeneration als grundlegende Existenzbedingung des ganzen Universums auf. Die Lebensdauer 
des Kosmos erweist sich hier als genauso begrenzt wie die Zeit, die als eine biologische Lebensform existieren kann. ${ }^{60}$

In der Erzählung geht die Degeneration der Menschheit, die Stück für Stück in einem traumlosen, komatösen Zustand versinkt, mit den erfolglosen Versuchen der Pflanzen- und Tierwelt einher, sich den veränderten Lebensbedingungen anzupassen; der Countdown des Universums, der auf die Erde gefunkt wird und dort in Form von Zahlenkolonnen, die Kaldren aufzeichnet, langsam das Ende aller Zeiten herunterzählt, generiert eine unbestimmbare Endzeitstimmung, die die Geschichte durchdringt und in den leeren Landschaften Tacita Deans widerhallt. Der Protagonist Powers nimmt am Ende der Geschichte, nachdem er selbst seine >schlafenden Gene aktiviert hat, einen Zustand an, in dem er die Zeit regelrecht fühlen kann und in dem sich ihm die Jahrmillionen des vor ihm aufgetürmten Bergmassivs und des prähistorischen Salzsees zugleich auditiv - als Stimmen der Zeit - und visuell - als mentales Bild - vermitteln: »a psychic picture defined and clear as the visual image brought to him by his eyes «. ${ }^{61}$ Solcherart von den Zeitzonen der ihn umgebenen Landschaft durchdrungen, kann er sein Auto mit geschlossenen Augen zwischen den zwei Zeitfronten des Sees und der Berge hindurchlenken:

\begin{abstract}
Closing his eyes, Powers lay back and steered the car along the interval between the two time fronts, feeling the images deepen and strengthen within his mind. The vast age of the landscape, the inaudible chorus of voices resonating from the lake and from the white hills, seemed to carry him back through time, down endless corridors to the first thresholds of the world. ${ }^{62}$
\end{abstract}

Powers selbst wird somit zu einem Medium, das die verschiedenen Zeiten in sich aufnimmt und zwischen ihnen vermittelt. Kaldren prophezeit an anderer Stelle: »Think of yourself in a wider context. Every particle in your body, every grain of sand, every galaxy carries the same signature. ${ }^{63}$ Ballard beschreibt in anschaulichen Bildern die Vergangenheit in Form des Alters der Landschaft, von der sein Protagonist jedes Sandkorn und jeden Salzkristall fühlen kann, die Zukunft des nahenden Untergangs des Universums und Powers körperliche Gegenwärtigkeit, die in das Kontinuum der Zeit eingebettet ist und schließlich

6o Michael K. Iwoleit, »Raum Zeit und Entropie. Eine Einführung in J. G. Ballards Kurzgeschichtenwerk«, in: Joachim Körber (Hg.), J. G. Ballard - Der Visionär des Phantastischen, Edition Futurum, Band 6, Meitingen 1985, S. 9-23, S. 15.

61 James Graham Ballard, The Voices of Time, London 1985, S. 38.

62 Ebd.

63 Ebd., S. 35 . 
in diesem aufgehen wird (als Powers stirbt), als Ebenen der Zeit, die letztlich gleichzeitig existieren, in direktem Zusammenhang stehen.

Diese Parallelisierung unterschiedlichster Zeitkonzepte und Materialisierungen bei Ballard greift Dean in ihrem Film auf, indem sie drei Einstellungen in einem Bild miteinander kombiniert. Es existiert keine Hierarchie zwischen den Aufnahmen der arbeitenden Maschinen, der großen Schaufelbagger und prähistorisch anmutenden Turbinen, die Salzwasser in Auffangbecken schaufeln, zwischen den Detailstudien von Salzkristallen im Wasser oder am Ufer, den großen Granitblöcken, der Geschäftigkeit des kleinen Gürteltiers und dem Licht der untergehenden Sonne über den Bergen. Die Mehrfachprojektion ebenso wie die collageartigen Bilder, die durch die Maskierungen entstehen, suggerieren eine Simultaneität der Zeiten, die auch Smithson in seinem Film Spiral Jetty durch die Kombination der unterschiedlichen Aufnahmen erreicht. ${ }^{64}$

Seine Entsprechung findet dieses Konzept der Parallelisierung durch Kontrastierung wiederum in Ballards nichtlinearer Erzähltechnik und seiner sehr bildhaften Sprache. Michael Iwoleit zieht einen Vergleich zur filmischen Montagetechnik, wenn er das Erzählen Ballards als »filmartige Technik des >nichtlinearen< Erzählens«65 beschreibt. Ballard habe sich bewusst vom linearen Erzählen abgewandt, da er fand, »daß die Handlungen und Ereignisse besonders der Romane beim Schreiben [...] in Teile zerfielen, daß sich die Charakterisierung, die Abfolge der Ereignisse zu einer Serie immer kürzer werdender Bilder und Situationen herauszukristallisieren begann «.66 Deans Film ist ähnlich aufgebaut: Die Abschnitte zwischen den Uhren werden immer kürzer, und ihre Bildsequenzen sind ebenso wenig linear und narrativ, sondern kreieren Korrespondenzen und Brüche zwischen Bildern.

Auch im Hinblick auf die Science-Fiction-Thematik sind die Korrespondenzen zwischen Smithson und Ballard augenfällig und werden von Dean in ihrem Film in Bilder übersetzt. Das Grundthema der Science Fiction - die mögliche Entwicklung unserer Zivilisation in einer oft nicht näher benannten

64 Zum Beispiel die dokumentarischen Aufnahmen der Erstellung der Mole, die Bilder der Sonne, Detailaufnahmen der Salze und der Wasseroberfläche, die Aufnahmen des Dinosaurierskeletts im naturhistorischen Museum, schließlich die abgefilmten Karten, die auf unterschiedliche historische Zeiten des Ortes und seiner Vermessung verweisen, Fotografien, die als Standbilder den Fluss des Films unterbrechen, und die schwindelerregenden Hubschrauberaufnahmen der Spirale.

65 Iwoleit, »Raum Zeit und Entropie«, S. 16.

66 »Die neue Science Fiction. Ein Gespräch zwischen J. G. Ballard und George MacBath«, in: Langdon Jones (Hg.), Neue SF. Eine Anthologie moderner spekulativer Literatur, Frankfurt am Main 1973, S. 57, zit. n. Iwoleit, »Raum Zeit und Entropie«, S. 16. 
Zukunft unter Berücksichtigung von wissenschaftlichen und technischen Neuerungen - hat Smithson während seiner gesamten künstlerischen Laufbahn interessiert; in zahlreichen seiner Texte finden sich Verweise auf Science-Fiction-Literatur, Naturwissenschaften und Astronomie. ${ }^{67}$ Samantha Schramm hat mit Bezug auf John Taines Roman The Time Stream darauf hingewiesen, dass sich das darin beschriebene Zeitkonzept eines Zeitstroms mit wechselnden Geschwindigkeiten und Richtungen auch auf den Film als Medium beziehungsweise auf Smithsons Film Spiral Jetty übertragen ließe. In jenem werde auch ein Wechsel unterschiedlicher Zeitlichkeiten inszeniert, »[d]ie Zeit von Spiral Jetty erscheint fragmentarisch, wird in ihren Brüchen und Diskontinuitäten ausgestellt«.68

In einem kurzen Text über die Entstehung von $J G$ verweist Dean auf die Parallele, die sie im Motiv der Spirale bei Smithson und Ballard gesehen hat einerseits in der mandalaartigen Skulptur im Salzsee, andererseits jedoch auf der symbolischen Ebene. ${ }^{69}$ Die Spirale ist für sie ein Symbol für die Zeit, und zwar eine analoge Zeit, wie sie sie für den Film als Medium propagiert und die sie in den Werken des Künstlers und des Autors wiederzufinden glaubt: »Both works have an analog heart, not just because they were made or written when spooling and reeling were the means to record and transmit images and sound, but because their spiraling is analogous to time itself. $\ll^{70}$ Die Spirale schafft in ihrer Wahrnehmung eine Verbindung zwischen der prähistorischen und der kosmischen Zeit, in deren Mitte die Spiral Jetty sozusagen als gigantische Uhr liegt; als `Zeitgestalt` durchdringt die Spirale die Zeitschichten der Vergangenheit, welche bei Smithson mit den Erdschichten bis zum Ursprung des früheren Sees gleichgesetzt werden, und weist auf der anderen Seite in den Kosmos und das Ende der Zeit, wie das Mandala in The Voices of Time:

While Smithson's jetty spiraled downward in the artist's imagination through layers of sedimentation and prehistory, in ancient repetition of a mythical whirlpool, coiling beneath the surface of the lake to the origins of time in the core of

67 Smithson besaß ein Exemplar von The Voices of Time und könnte sich für Spiral Jetty an der Betonskulptur, die Powers errichtet, orientiert haben. Dem Text »Der Künstler als Orts-Seher. Ein dintorphischer Essay« stellt er eine Passage aus Ballards The Terminal Beach voran. In dem im Film Spiral Jetty gezeigten Bücherstapel findet sich auch Arthur Conan Doyles Die vergessene Welt, und im Epigraf zu »Entropie und Neue Monumente« zitiert Smithson aus John Taines The Time Stream.

68 Samantha Schramm, Land Art. Ortskonzepte und mediale Vermittlung zwischen Site und Non-Site, Berlin 2014, S. 183.

69 Vgl. Dean, $\gg \mathrm{JG} \ll$.

70 Ebd. 
the earth below, the mandala in >The Voices of Time < is its virtual mirror, kaleidoscoping upwards into cosmic integration and the tail end of time. ${ }^{71}$

Die symbolische Form der Spirale ist so auch ein Bild für eine Zeitstruktur, welche sich in beide Richtungen aufwinden lässt wie ein Film. Die Gleichsetzung des Films mit einer Spirale, die Smithson formuliert (»ein Film ist eine Spirale aus Bildern ${ }^{72}$ ), überträgt Dean auf ihre Wertschätzung des Mediums, das sie an anderer Stelle mit der Zeit gleichgesetzt hatte.

\section{Kubler, Form der Zeit}

An die Zeitlichkeit der Entropie bei Smithson und Ballard anschließend, soll nun noch ein Blick auf ein kunsttheoretisches Modell der Zeit geworfen werden, das oben bereits angesprochen wurde und für Robert Smithson und andere Künstler in den 196oer Jahren Bedeutung erlangte: George Kublers Form der Zeit. Das Buch erschien 1962 und wurde vom Smithson und anderen Künstlern wie Norbert Weiner und John Baldessari stark rezipiert, obgleich Kubler sich nicht mit europäischer Moderne, sondern mittelamerikanischer Kunst der Kolonialzeit und früher beschäftigt. ${ }^{73}$ Im Gegensatz zur konventionellen Kunstgeschichte betreibt Kubler in seinem Essay eine Kulturgeschichte, die nicht zwischen Kunstwerken und anderen, insbesondere wissenschaftlichen Dingen unterscheidet, sondern die zeitliche und historische Verortung aller Artefakte unter dem »Oberbegriff der visuellen Form « ${ }^{74}$ vornimmt.

In mehreren Texten setzt sich Smithson explizit mit Kubler auseinander: In »Quasi-Unendlichkeiten und das Schwinden des Raumes « ${ }^{75}$ beschreibt er, wie sich Kublers Zeitvorstellung, besonders die Rolle der >Aktualität<, in den abstrakten Black Paintings von Ad Reinhardt wiederfindet. Nach Kubler ist die einzig unmittelbar wahrnehmbare Zeiteinheit die des >Jetzt<, der Aktualität, was sich für den Historiker als Problem darstellt: »Die übrige Zeit macht sich nur in Signalen bemerkbar, die uns in diesem Augenblick auf unzähligen Ebenen übermittelt werden und von ganz unerwarteten Sendern. «76 Die Aktualität ist einerseits ein ungreifbar kleines Intervall, die »Leere zwischen

\footnotetext{
71 Ebd.

72 Smithson, »Spiral Jetty«, S. 18o.

73 Zur Rezeption Kublers in der Kunst der 196oer Jahre vgl. Pamela M. Lee, Chronophobia. On Time in the Art of the 196o's, Cambridge, MA 2004, Kapitel 4: »Ultramoderne: Or, How George Kubler Stole the Time in Sixties Art«, S. 218-256.

74 Kubler, Die Form der Zeit, S. 42.

75 Robert Smithson, »Quasi-Unendlichkeiten und das Schwinden des Raumes« (1966), in: ders., Gesammelte Schriften, S. 54-57.

$76 \quad$ Kubler, Die Form der Zeit, S. 51.
} 
den Ereignissen «, 77 andererseits ist nur im Jetzt die Möglichkeit gegeben, das Damals in Form von Signalen aus der Vergangenheit, historischen Indizes, zu begreifen. In einem treffenden Vergleich zieht Kubler Parallelen zwischen dem Historiker und dem Astronomen: Sie beschäftigen sich beide mit der Darstellung von Zeit, sehen aber beide nur »altes Licht«, die Signale längst vergangener Ereignisse: »[S]ie befassen sich beide mit Erscheinungen, die sie in der Gegenwart wahrnehmen, die aber in der Vergangenheit geschehen sind. « ${ }^{78}$ Diesem Gedanken liegt ein diskontinuierliches Verhältnis von Zeitlichkeit und Geschichtlichkeit zugrunde, denn der historische Moment wird von Kubler innerhalb der Sequenz einer Problemlösungsreihe verortet, innerhalb derer Artefakte in einem nicht notwendig chronologischen Verhältnis zueinander stehen. Für Smithson und seine Zeitgenossen war darüber hinaus von Interesse, dass Kubler sich mit Blick auf die Übertragung von Energien in der Kunst an Begriffen der Physik und Informationstechnologie (Impulse, Signale, Relais) orientiert und nicht an Metaphern aus der Biologie. Kubler kritisiert die in der Stilgeschichte vorherrschenden biologischen Entwicklungsmodelle Typologie und Morphologie, da sie der Möglichkeit finalen Denkens entbehrten und stattdessen von ständig wiederkehrenden Prozessen ausgingen: »Finales Denken hat keinen Platz in der Biologie, Geschichte jedoch ist ohne das sinnlos. «79 Er beschreibt im Epilog seiner Abhandlung in Bezug auf die formalen Entwicklungsmöglichkeiten der Kunst das Szenario einer »finiten Welt«:

Wir müßten erkennen, daß wir uns nicht in einem expandierenden Universum von Formen befinden, wie die zeitgenössischen Künstler fest, aber voreilig annehmen, sondern daß wir eine finite Welt mit begrenzten Möglichkeiten bewohnen, die immer noch weitgehend unerforscht ist und Abenteuern und Entdeckung weiterhin offensteht. Sie ist den Polarwüsten vergleichbar, lange bevor Menschen dort Fuß gefaßt haben. ${ }^{80}$

Diese Begrenztheit der Welt liegt dem Gedanken der Entropie zugrunde und wirkt sich für Kubler auch auf die Rolle der Zeit aus: Wir steuerten eben nicht auf eine unbegrenzte Zukunft zu, vielmehr sei diese in ihren Möglichkeiten der Veränderung begrenzt und immer schon von der Vergangenheit vorgeprägt.

So, wie Smithson sich in seiner Geschichtsauffassung an Kubler orientiert, weist auch Deans CEuvre in der Darstellung von Geschichte Parallelen zur Konzeption von Kubler auf. In ihren Werken agieren Dinge, Menschen und

\begin{tabular}{ll}
\hline 77 & Ebd. \\
78 & Ebd., S. 54. \\
79 & Ebd., S. 40. \\
80 & Ebd., S. 196.
\end{tabular}


Orte als Zeugen der Vergangenheit, deren Repräsentation auf Film die Tatsache, dass sich ihrer erinnert wird, unterstreicht. Dabei ist die Welt, die sie repräsentiert, eine des Alters, der Ruinen, des Vergessens. Viele Bauwerke, die sie darstellt, verweisen durch ihren historischen, häufig politischen Hintergrund auf eine spezifische vergangene Zeit, die ihre Signale aus der Vergangenheit in die Gegenwart sendet und deren bildliche Darstellung im zeitbasierten Medium Film die Erfahrung dieses historischen Verweises erlebbar macht.

$J G$ zeigt sich einerseits als Film, der mit den Mitteln der filmischen Montage und Collage die Gemeinsamkeiten von Smithsons und Ballards Werken und ihres jeweiligen Geschichts- und Zeitverständnisses analysiert. Hierfür greift Dean Motive wie die Turbinen und Baumaschinen, die Echsen und die Salzkristalle auf und generiert in der Kombination der Bilder und der gesprochenen Zitate einen Dialog zwischen den beiden Künstlern und ihren Werken. Andererseits geht $J G$ weit über eine simple Zusammenschau von Spiral Jetty und The Voices of Time hinaus. Deans menschenleere Szenerien, die sich wie automatisiert bewegenden Maschinen mit unklarem Arbeitsauftrag, die wuchernden Salzkristalle, Ausblicke auf den Salzsee und weite Berglandschaften wenden nämlich den Blick in die Zukunft: Sie schaffen das Szenario einer Welt nach dem Ende der Menschheit, einen dystopischen, heterotopischen Raum, in dem die Zeit stillzustehen scheint, weil kein menschliches Maß mehr an ihr Verstreichen angelegt wird. In dieser »finalen Welt« sind die Überbleibsel der Zivilisation sich selbst überlassen, wie es oft in Deans Filmen zu beobachten ist: Man denkt an Teignmouth Electron (1999), Bubble House (1999) und Sound Mirrors (1999), die als ruinenhafte Hinterlassenschaften von menschlichen Schicksalen, ästhetischen und technischen Entwicklungen sowie politischen Entscheidungen zeugen. Zu JG gehören so auch Artefakte, die sie salinisiert hat (ihre Ausgabe von The Voices of Time zum Beispiel, die, über und über mit Salzkristallen bedeckt, unlesbar geworden ist). In $J G$ sind es erneut die langen, beobachtenden Einstellungen, welche die Zeitlichkeit des Bildes greifbar werden lässt; sie machen für die Zuschauer/ innen die Filmzeit in Bezug zur Realzeit bewusst. Wie oben beschrieben, hält ihre Länge und »Losigkeit « 81 , wie es Achim Hochdörfer genannt hat, sie genau in der Schwebe zwischen montierter, narrativer Einstellung und einem als einzelnes wahrgenommenen Bild.

$J G$ zeigt aber auch in anschaulichen Bildern die Ablagerungen beziehungsweise, in der Sprache Foucaults, die Akkumulation von Zeit in Materialien, in Stein, Salz und Erde, die in zahlreichen Schichten die geologische Zeit symbolisieren. Das Gürteltier wie auch die Maschinen graben darin, schichten

81 Hochdörfer, »Tacita Dean Makes a Film«, S. 41. 
die Steine auf und um und sind so die Arbeiter am Steinbruch der geschichtlichen Zeit, deren Sedimente so alt sind und die die Menschen trotzdem überdauern werden. Tacita Deans Film schafft ein Bild von einer Landschaft, für die die Interventionen der Menschen nur ein kurzes Gastspiel bedeuten, wie auch Robert Smithson erkannt hat. Seine Spiral Jetty verbindet den historischen Ort des Sees mit einer mythischen Form; Deans Film zeigt den Ort als Zeitspeicher, in dem die verschiedenen Zeitrechnungen - geologische Zeit, kosmologische Zeit, Zeit des Betrachters, Zeit des Films - zusammentreffen. 